\title{
The Relationship Between Anxiety, Depression and Treatment Adherence in Chronic Obstructive Pulmonary Disease: A Systematic Review
}

\author{
Eleonora Volpato $\mathbb{D}^{1,2}$ \\ Stefania Toniolo' \\ Francesco Pagnini $\mathbb{1}^{1,3}$ \\ Paolo Banfi $\left(\mathbb{D}^{2}\right.$ \\ 'Department of Psychology, Università \\ Cattolica del Sacro Cuore, Milan, Italy; \\ ${ }^{2}$ Heart-Respiratory Rehabilitation Unit, \\ IRCCS Fondazione Don Carlo Gnocchi, \\ Milan, Italy; ${ }^{3}$ Department of Psychology, \\ Harvard University, Cambridge, MA, USA
}

Correspondence: Eleonora Volpato Email eleonora.volpato@unicatt.it
Background: Almost half of the people with chronic obstructive pulmonary disease (COPD) do not adhere to the prescribed treatments and report anxiety and depression as comorbidities, resulting in higher rates of exacerbations, hospitalizations, and worse clinical outcomes.

Objective: This systematic review provided a synthesis of studies about the relationships between anxiety, depression, and adherence in people affected by COPD.

Methods: English language publications were searched in the PUBMED, SCOPUS, PsycInfo, Web of Science, PsycArticles, and Cochrane Library databases from December 2020 to March 2021, following PRISMA guidelines. The reference lists of eligible studies and other relevant systematic reviews were also searched. Data extraction and critical appraisal were undertaken by two reviewers working independently. The reference lists of eligible studies and other relevant systematic reviews were also searched. Data extraction and critical appraisal were undertaken by two reviewers working independently.

Results: A total of 34 studies (23 quantitative and 2 qualitative studies, 9 reviews) were included. The relationship between depression and treatment adherence was significant and negative. Adherence to both rehabilitation, psychological, and antidepressant pharmacological treatments in depressed patients was linked to a decreased risk of hospitalization. Moreover, depressed patients compliant with an antidepressant were more likely to adherent to COPD maintenance inhalers. On the other hand, the associations between anxiety and adherence were poorly investigated and high heterogeneity characterized the studies, leading to a weak and variable relationship as well as too few interventions.

Conclusion: The systematic review highlights the variability in estimates of the relationship between depression, anxiety, and treatment adherence in COPD. It could be explained by methodological differences across the included studies. This suggests that standardization is critical to improving the precision of the estimates. Recommendations for future research include attention to causal inferences, an exploration of mechanisms to explain the relationships between both anxiety and depression and adherence in COPD, and a comprehensive, systematic approach.

Keywords: chronic obstructive pulmonary disease, adherence, compliance, anxiety, depression, systematic review

\section{Introduction}

Chronic Obstructive Pulmonary Disease (COPD) is an irreversible but treatable condition characterized mostly by dyspnoea, cough, and sputum production. Tobacco smoking is the main exposure but air pollution, genetic or abnormal lung development may contribute. ${ }^{1}$ It is the fourth cause of morbidity worldwide and it is expected to be soon the third leading cause of mortality. ${ }^{2,3}$ 
Comorbidity with anxiety and depression is frequent and well known for quite some time ${ }^{4,5}$ although often underrated due to the priority of symptoms management, ${ }^{6,7}$ especially when coexisting with physical illness. ${ }^{8}$ The Global Initiative for Chronic Obstructive Lung Disease (GOLD) emphasizes the importance of anxiety and depression assessment as a part of the evaluation of COPD patients. ${ }^{7}$ Prevalence and incidences' rates vary widely across studies, owing to differences in sampling methods and degrees of illness severity as well as in assessment instruments adopted. The reported prevalence of depression in COPD ranges from $10 \%$ to $42 \%$ in people with stable COPD and from $10 \%$ to $86 \%$ in those with an acute exacerbation. Prevalence rates for clinical anxiety in COPD range from $13 \%$ to $46 \%$ in outpatients and $10 \%$ to $55 \%$ among inpatients. ${ }^{9}$ According to Biswas et al, ${ }^{7}$ the prevalence of depression and anxiety varies from $12 \%$ to $57 \%$ in different studies from western countries. Existing evidence suggests that there is an increasing prevalence of anxiety disorders in COPD patients ${ }^{10}$ varying from $2 \%$ to $50 \%,{ }^{11}$ and as many as $55 \%$ of these patients may suffer from psychiatric disorders. ${ }^{12}$ The most common depressive disorder in COPD is represented by major depressive disorder, followed by dysthymias (chronic depressive symptoms of mild severity), and minor depression. On the other hand, generalized anxiety disorder (GAD), phobias, and panic disorders mainly occur in the clinical picture of anxiety. It is also relevant to stress that depression and anxiety often occur simultaneously in patients with COPD, ranging from $26 \%$ to $43 \%$. Depression and anxiety could also interact with other risk factors (like smoking) to produce stronger combined effects on mortality. ${ }^{2}$

Anxiety and depressive symptomatology have an impact on COPD exacerbations ${ }^{13}$ and mortality. ${ }^{14,15}$ Anxiety is negatively associated with exercise performance ${ }^{16}$ while depression does not seem to be significant in predicting walk distance of COPD patients. ${ }^{7}$ Depression is associated with lower health-related quality of life ${ }^{17}$ and higher hospitalizations. ${ }^{14}$ Growing evidence suggests that the relationship between anxiety, depression, and COPD is bidirectional. Therefore, they adversely impact prognosis in COPD as well as COPD increases the risk of developing depression and/or anxiety. Disease symptoms are, in fact, important determinants of depression in COPD, and anxiety and depression worsen COPD outcomes, triggering a vicious circle. ${ }^{15,18}$ Nevertheless, the exact relationship between anxiety, depression, and COPD remains unclear. $^{18}$ More longitudinal studies are required. ${ }^{15}$ For example, a longitudinal analysis ${ }^{19}$ identified depression as a risk factor for breathlessness, and not vice versa. Study ${ }^{20}$ followed 1580 COPD patients in a 3-years follow-up, finding that about one in four had persistent depressive symptoms and those experienced more exacerbations and loss in performance. Anxiety and depression are more closely related to Health-Related Quality of Life (HRQoL) than to lung function parameters, ${ }^{21}$ even though they are strongly related to dyspnoea ratings both after exercise and during everyday activities. $^{22}$ Health outcomes are worse in COPD people with mood disorders, although the exact mechanism for the association remains unclear. ${ }^{23}$

Anxiety and depression can interfere with COPD management, creating a pathologic cycle of deteriorating health status, ${ }^{15}$ and lowering adherence to treatments and pulmonary rehabilitation. ${ }^{15,24,25}$ Being a treatable disease, patient self-management, adherence, and self-care behavior are important aspects of COPD treatment. ${ }^{26}$ Although anxiety, depression, and treatment adherence have been studied intensively in COPD, less is known about the association between these factors. According to study, ${ }^{27}$ for example, depressed COPD patients are 3 times more likely to be non-compliant with prescribed medication but also exercise, diet and health-related behavior, but few studies quantified the impact of anxiety or depression on adherence or vice versa.

The present systematic review provides an essential update of the expanding literature characterizing the relationship between anxiety, depression, and treatment adherence in patients with COPD. This paper also aims to integrate evidence to examine whether these highly comorbid conditions share underlying features and to critically appraise the effect of methodological inconsistencies and confounding factors. A more nuanced understanding of the pathophysiology of anxiety and depressive disorders and their relationship with adherence may inform future diagnosis and treatment options in COPD.

\section{Methods}

The protocol was registered with PROSPERO international prospective register of systematic reviews (\# CRD42021238035).

\section{Search Strategy, Eligibility Criteria, and Information Sources}

Systematic searches were conducted from December 2020 to March 2021 following PRISMA guidelines ${ }^{28}$ (Appendix 
B). The PUBMED, SCOPUS, PsycInfo, Web Of Science, PsycArticles, and Cochrane Library databases were consulted to capture human studies from inception to $25^{\text {th }}$ March 2021 that: specified a relationship between depression and/or anxiety and adherence/compliance in COPD patients: studies with populations with other illnesses beside COPD (eg, asthma) were included only if the results for the COPD group were highlighted and separated from other groups; considered the patient's point of view, and their experience, not the physicians or caregiver's.

Inclusion and exclusion criteria, as well as comprehensive search terms, are presented in Appendix A.

\section{Study Selection}

Titles, abstracts, and keywords were independently reviewed by two authors (EV; ST) excluding those that did not meet the eligibility criteria. A third author (FP) was consulted to resolve inconsistencies in screening decisions. Other articles were excluded because they were not available or not published. Full-text articles assessed for eligibility were read by the researchers and assessed following PICO criteria and inclusion/exclusion criteria.

\section{Data Extraction and Analysis}

Data were extracted by two independent researchers (EV; ST) and confirmed by a third one (FP). Information gathered from each study considered the following data: title, authors, publication year, country, journal, kind of study, demographic description of participants (gender, mean age, kind of illness, mean time from diagnosis), physiological parameters, family members, objectives of the study, outcomes, setting, follow-up, drop-out rate, inclusion and exclusion criteria, analysis, instruments, explored themes and findings. Data from the final data extraction forms were tabulated and are discussed in this review in a purely descriptive fashion. Due to the heterogeneity of the data and the diversity of the clinical trial designs, no additional sub-analyses or metaanalyses were planned or performed.

\section{Quality Assessment of Studies}

Methodological quality assessment of the included studies was completed with the Revised Cochrane risk-of-bias tool for randomized trials (RoB 2) ${ }^{29}$ for RCTs and the NIH quality assessment tool (https://www.nhlbi.nih.gov/ health-topics/study-quality-assessment-tools) for the other designs. The Critical Appraisal Skills Programme (CASP) qualitative checklist (https://casp-uk.net/wp-content
/uploads/2018/01/CASP-Qualitative-Checklist-2018.pdf)

was used for the qualitative studies.

\section{Results}

\section{Study Selection}

The search strategy allowed to identify 1136 potentially relevant records 304 articles were retrieved from PubMed, 292 from Scopus, 178 from Web of Science, 38 from PsycInfo, 214 from PsycArticles, 103 from Cochrane Library, and 7 from additional records (Figure 1). After removing 670 duplicates, a total of 466 records' titles and abstracts were screened and 115 were selected for full-text review. After removing the other 81 articles, 34 studies were included in this review. The most common reasons for exclusion were: no mention of anxiety and depression or adherence or no reference to the relationship between these variables.

\section{Study Characteristics}

The 34 included studies represent 15 different countries, including 16 from the USA, 3 from the UK, 3 from Italy, and 12 other countries (see Tables 1-3). Most of the studies were conducted in university settings or pulmonary rehabilitation centers (sometimes university-affiliated hospitals) and communities. Many studies, being retrospective, observational, cohort, etc., were conducted through databases or data collected in previous studies: Health Search Database, ADCARE study, Centers for Medicare, and Medicaid Services Chronic Condition Warehouse (CCW) data, CASCADE study, etc.

Twenty-three studies adopt a quantitative approach, 2 are qualitative and 9 are reviews. One of the 2 qualitative studies is an intervention description with a case example,${ }^{30}$ the other is an observational study with a phenomenological approach. ${ }^{31}$ Five studies are Randomized Controlled Trials, ${ }^{32-36}$ two ${ }^{37,38}$ are retrospective cohort studies. Four ${ }^{39-42}$ are cohort studies with a longitudinal approach. Two ${ }^{43,44}$ are defined as "retrospective longitudinal cohort studies". Seven ${ }^{25,45-50}$ are observational cross-sectional studies. One is a retrospective observational study. ${ }^{51}$ One is a retrospective cross-sectional study ${ }^{52}$ and one is defined as a "cross-sectional multicentre study". ${ }^{33}$

The 25 non-review studies represent a total of 153.105 adults with COPD. The mean age was between 60.4 and 75 years, with a range from 40 to 90 years. On average, females comprised from $7.9 \%$ to $76.5 \%$ of the study samples. In 15 

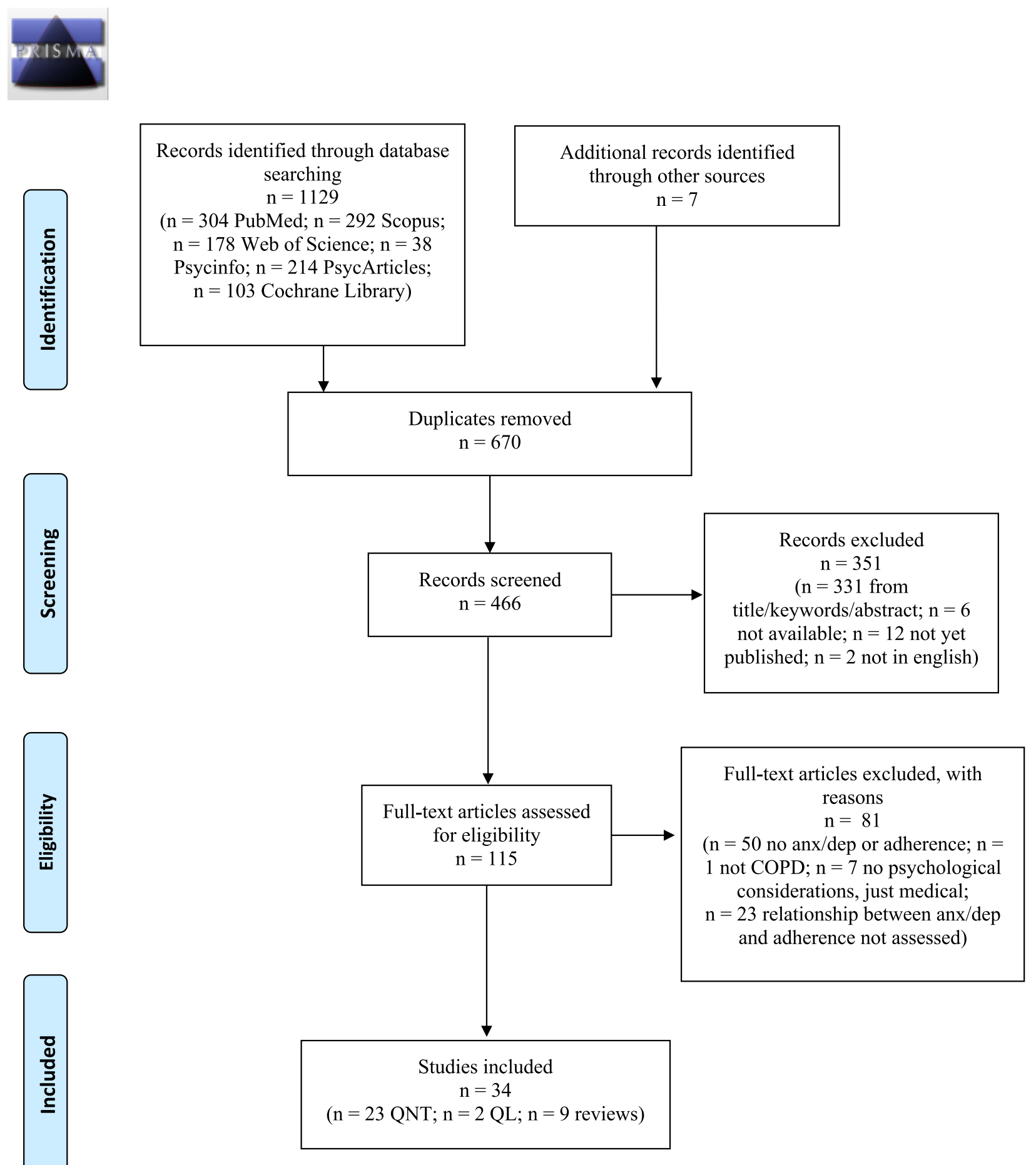

Figure I Flow chart diagram of the identification and selection of studies.

Notes: Adapted from: Moher D, Liberati A, Tetzlaff J, Altman DG, The PRISMA Group. Preferred Reporting Items for Systematic Reviews and Meta-Analyses: The PRISMA Statement. PLoS Med. 2009;6(7): el000097. 10.1371/journal.pmed I000097. ${ }^{28}$ For more information, visit www.prisma-statement.org.

of the 24 studies with sample description, females are more than males. Thirteen of 25 studies had a follow-up, mean follow-up time varied from 4 weeks to 24 months.
Sample sizes among studies ranged from 5 to 112 patients in the QL (qualitative) studies and from 39 to 74,863 patients in the QNT (quantitative) studies. 


\begin{tabular}{|c|c|c|c|c|c|}
\hline 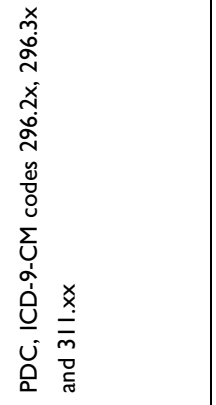 & 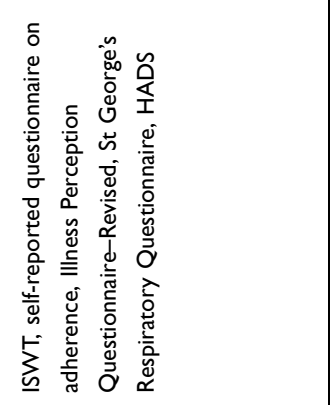 & 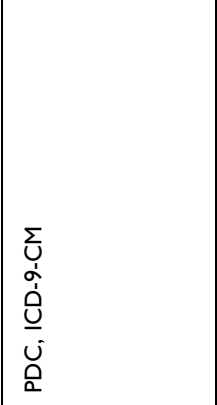 & 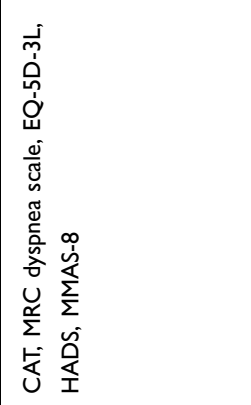 & 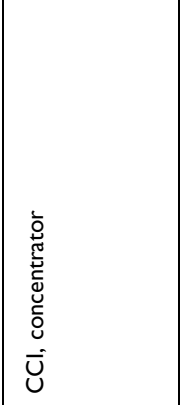 & 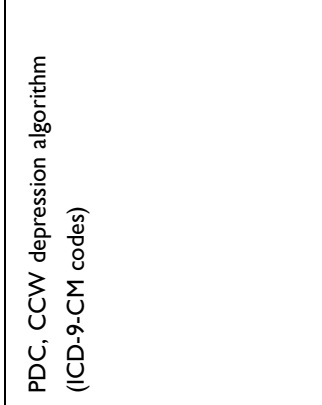 \\
\hline 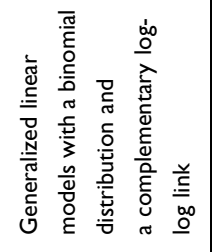 & 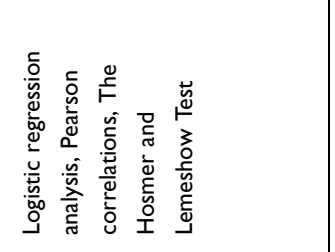 & 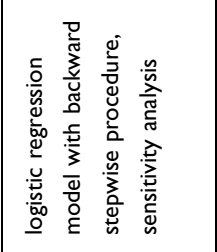 & 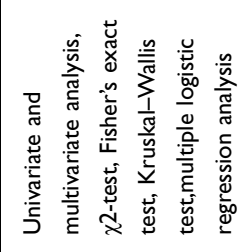 & 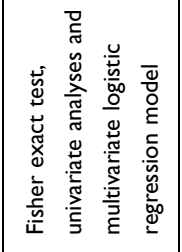 & 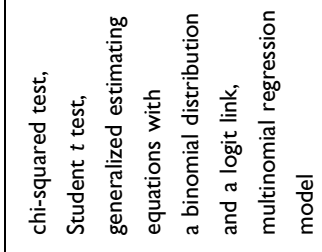 \\
\hline $\begin{array}{l}\text { U } \\
\frac{\mathrm{Z}}{\mathrm{W}} \\
\frac{\mathrm{W}}{\Sigma}\end{array}$ & 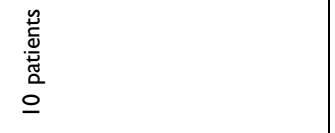 & $\begin{array}{l}\text { U } \\
\underline{\underline{W}} \\
\frac{W}{\Sigma}\end{array}$ & z & z & 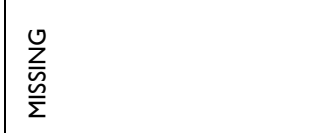 \\
\hline 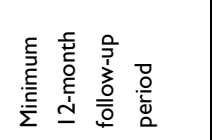 & 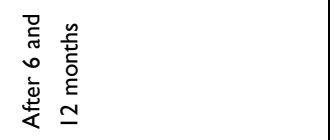 & 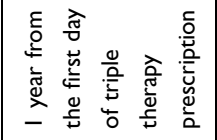 & 2 & 2 & 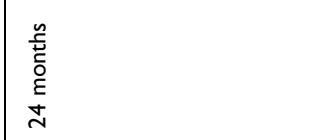 \\
\hline 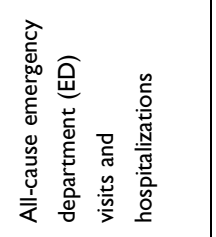 & 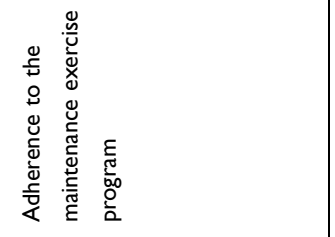 & 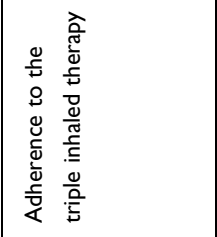 & 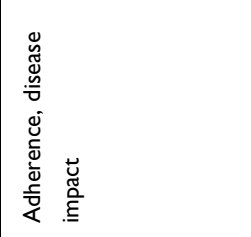 & 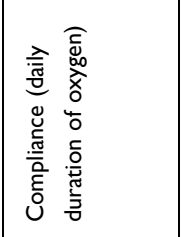 & 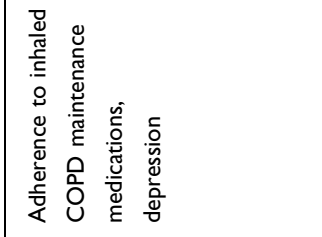 \\
\hline 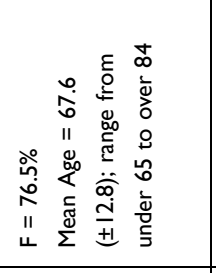 & 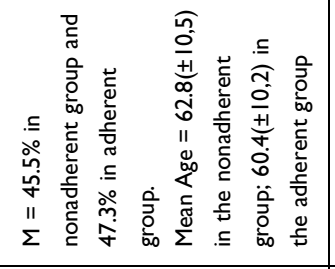 & 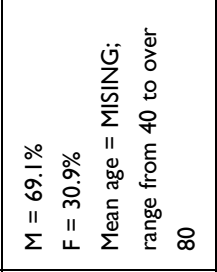 & 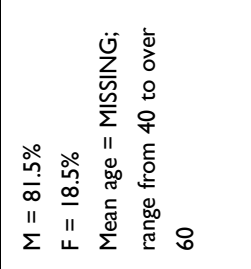 & 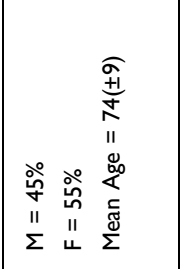 & 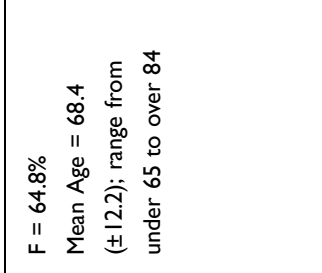 \\
\hline $\begin{array}{l}\hat{\hat{0}} \\
\underline{0}\end{array}$ & 尺 & $\frac{\mathrm{N}}{\mathrm{m}}$ & 光 & $\stackrel{\underline{n}}{=}$ & 蔡 \\
\hline 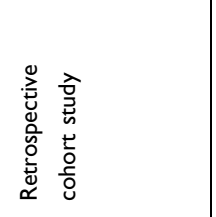 & 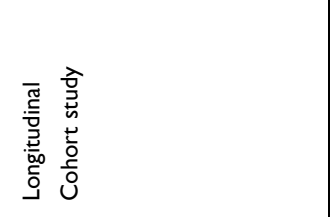 & 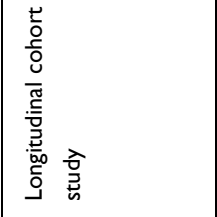 & 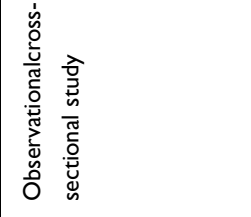 & 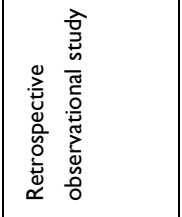 & 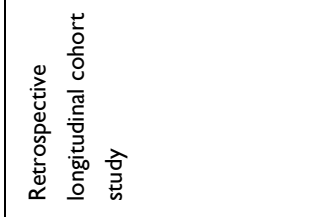 \\
\hline 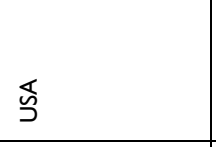 & 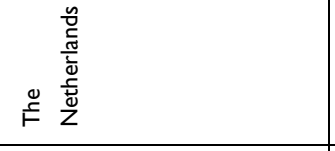 & ज्ञ & 岁 & 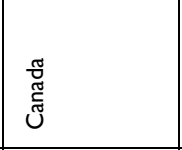 & 乌ీ \\
\hline 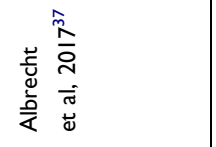 & 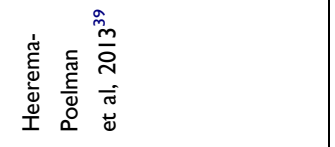 & 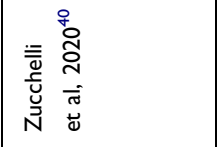 & 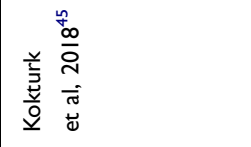 & 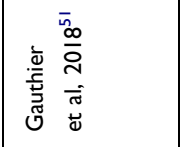 & 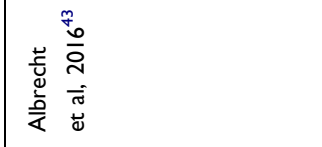 \\
\hline
\end{tabular}




\begin{tabular}{|c|c|c|c|c|c|c|}
\hline 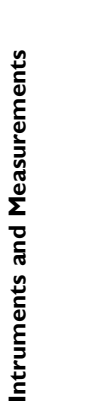 & 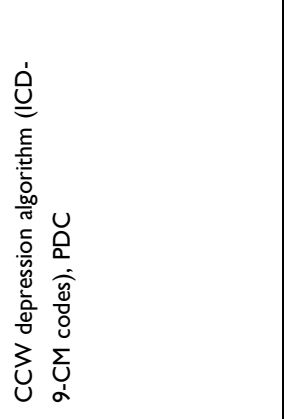 & 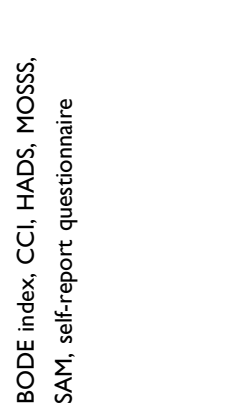 & 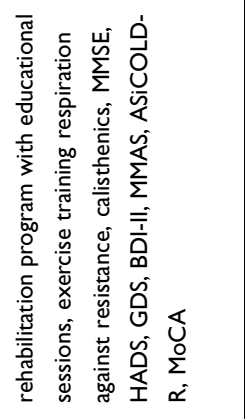 & 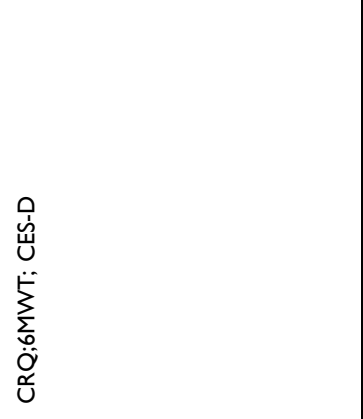 & 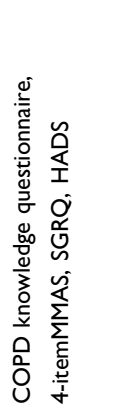 & 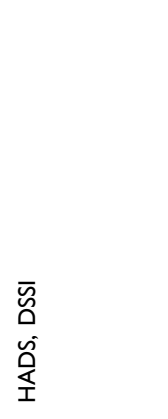 \\
\hline 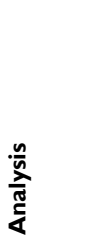 & 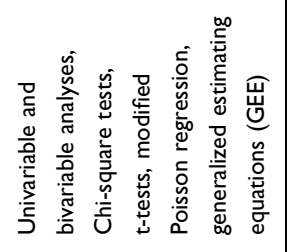 & 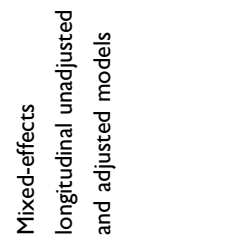 & 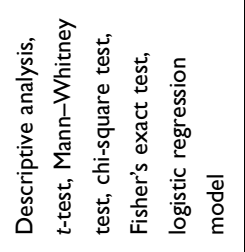 & 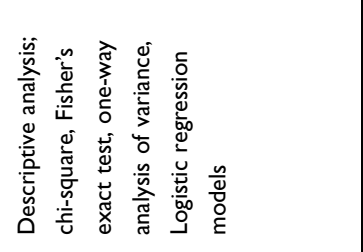 & 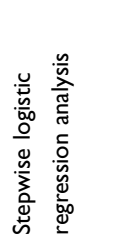 & 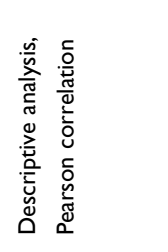 \\
\hline $\begin{array}{l}\text { ثे } \\
0 \\
\vdots \\
\vdots\end{array}$ & $\stackrel{\circ}{z}$ & 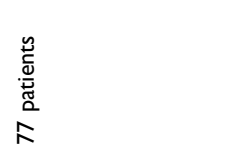 & $\stackrel{\circ}{z}$ & $\stackrel{\circ}{z}$ & zo & zo \\
\hline $\begin{array}{l}0 \\
\vdots \\
\vdots \\
\overline{0} \\
i\end{array}$ & $\stackrel{\circ}{z}$ & 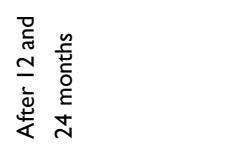 & $\stackrel{\circ}{z}$ & $\stackrel{\circ}{z}$ & $\stackrel{0}{z}$ & $\stackrel{0}{z}$ \\
\hline 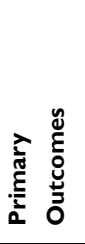 & 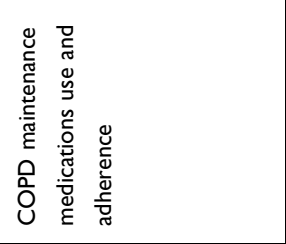 & 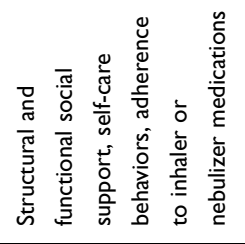 & 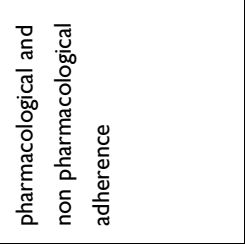 & 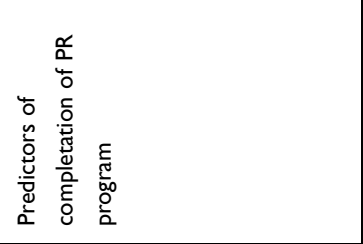 & 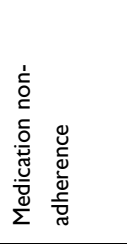 & 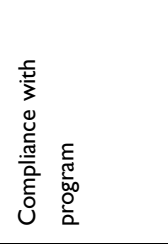 \\
\hline 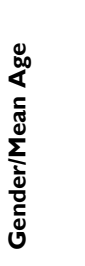 & 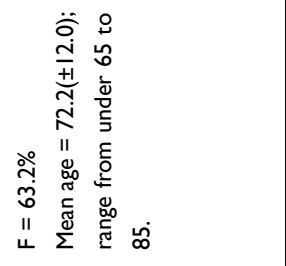 & 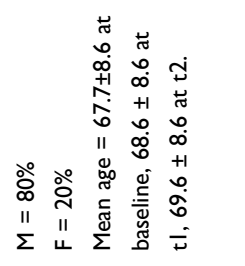 & 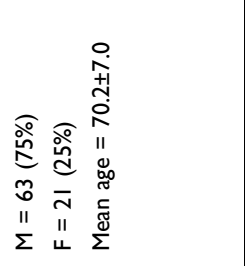 & 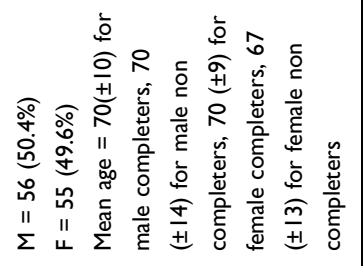 & 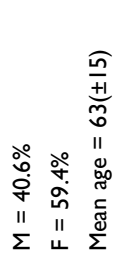 & 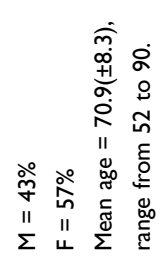 \\
\hline 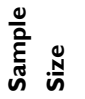 & 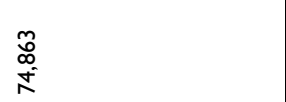 & $\underset{\sim}{\sim}$ & 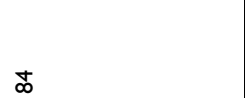 & $\equiv$ & $\underline{\underline{m}}$ & $\underline{\underline{n}}$ \\
\hline 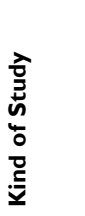 & 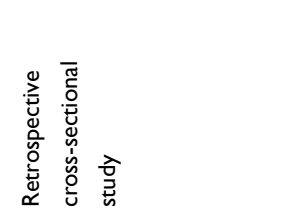 & 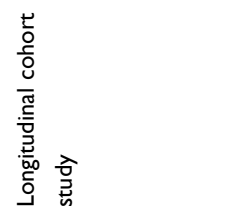 & 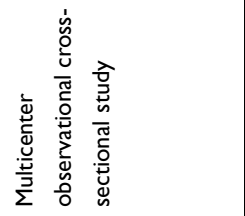 & 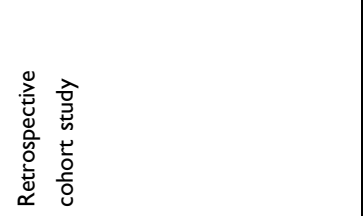 & 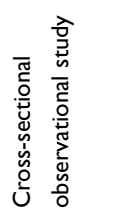 & 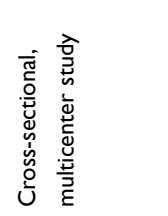 \\
\hline نे & 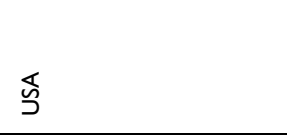 & 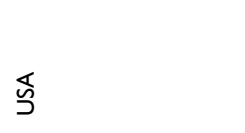 & 焉 & $\overleftrightarrow{\overleftarrow{s}}$ & 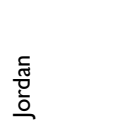 & 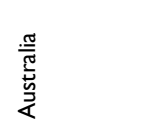 \\
\hline 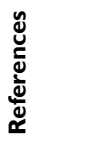 & 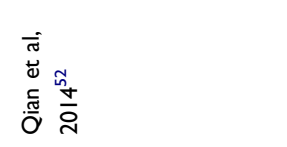 & 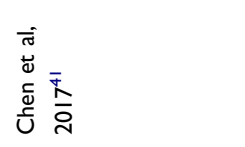 & 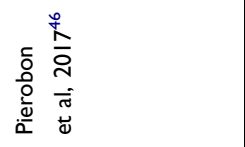 & 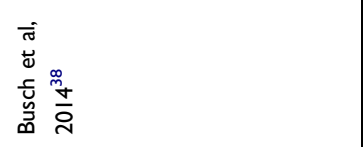 & 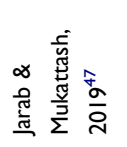 & 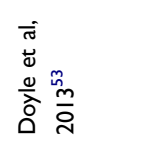 \\
\hline
\end{tabular}




\begin{tabular}{|c|c|c|c|c|c|c|}
\hline 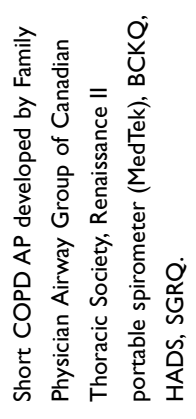 & 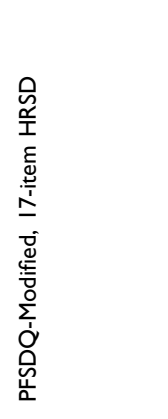 & 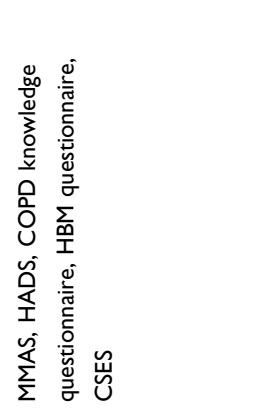 & 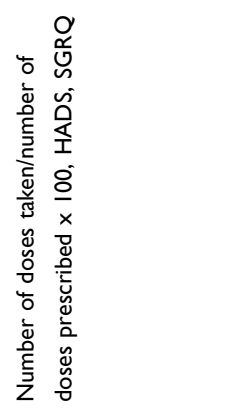 & 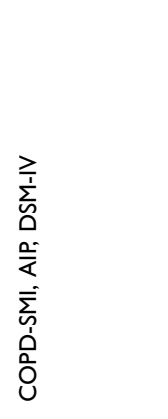 & 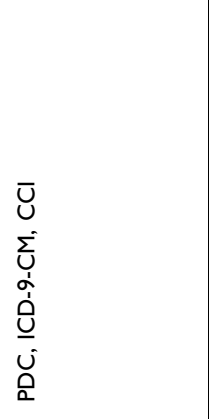 & 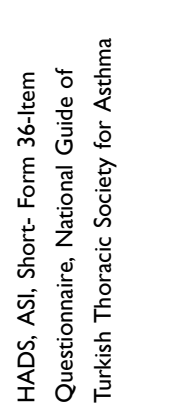 \\
\hline 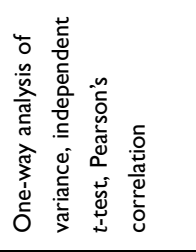 & 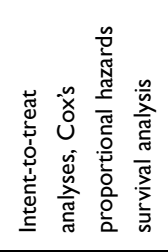 & 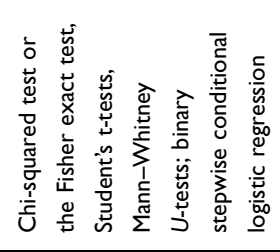 & 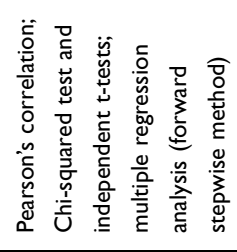 & 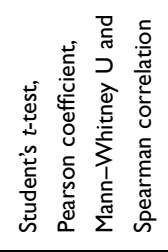 & 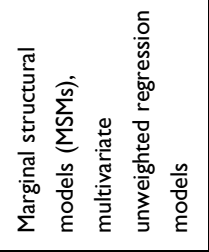 & 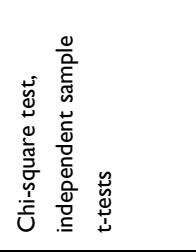 \\
\hline$\stackrel{\circ}{z}$ & 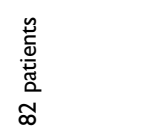 & $\stackrel{\circ}{z}$ & 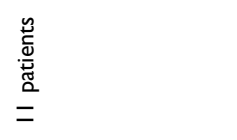 & $\stackrel{0}{z}$ & 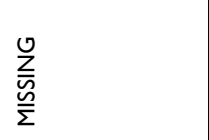 & 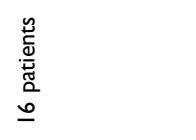 \\
\hline zo & 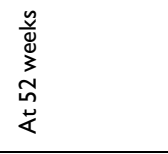 & $\stackrel{0}{z}$ & 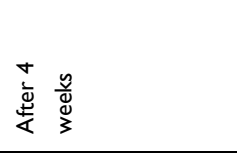 & $\stackrel{0}{z}$ & 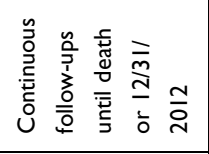 & 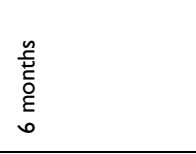 \\
\hline 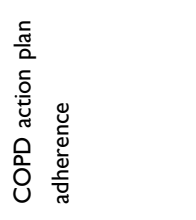 & 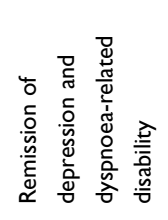 & 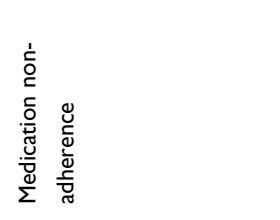 & 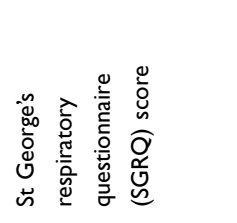 & 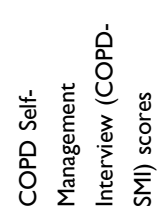 & 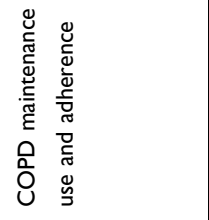 & 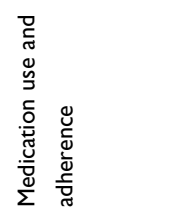 \\
\hline 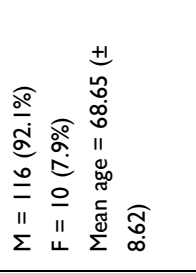 & $\begin{array}{l}\frac{U}{Z} \\
\frac{\underline{Z}}{\Sigma} \\
\frac{W}{\Sigma}\end{array}$ & 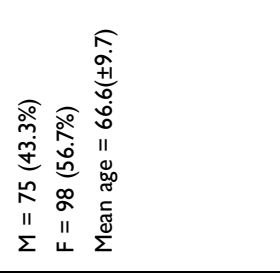 & 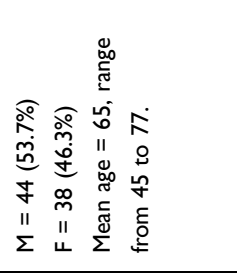 & 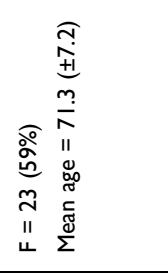 & 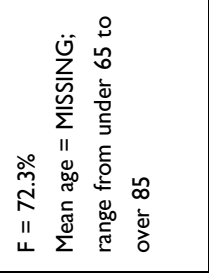 & 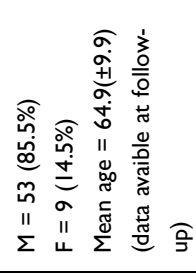 \\
\hline$\stackrel{ْ}{\simeq}$ & $\stackrel{\infty}{\underline{m}}$ & $\underline{\underline{M}}$ & $\widetilde{\infty}$ & 㝏 & 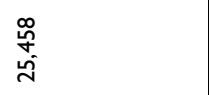 & $\stackrel{\infty}{\curvearrowright}$ \\
\hline 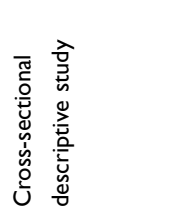 & 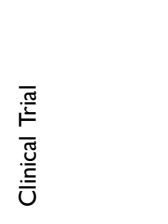 & 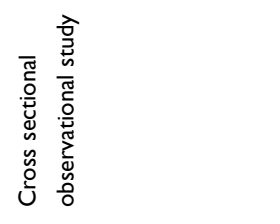 & 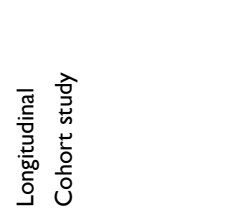 & 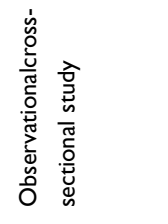 & 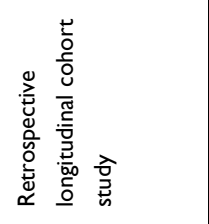 & 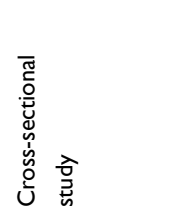 \\
\hline $\begin{array}{ll}\frac{\pi}{5} \\
\text { 离 } \\
0\end{array}$ & 芯 & $\stackrel{\text { J }}{ }$ & 弚 & 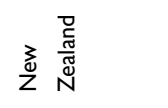 & 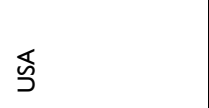 & 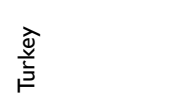 \\
\hline 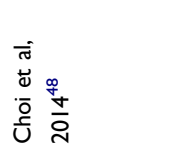 & 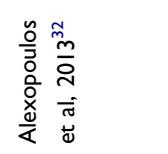 & 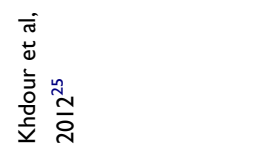 & 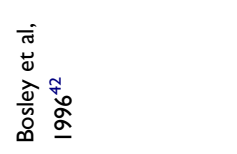 & 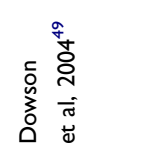 & 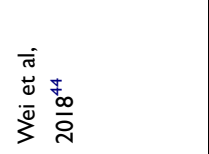 & 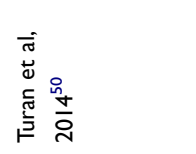 \\
\hline
\end{tabular}




\begin{tabular}{|c|c|c|c|}
\hline 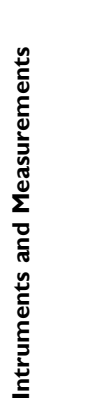 & 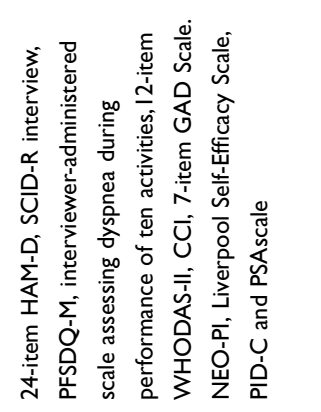 & 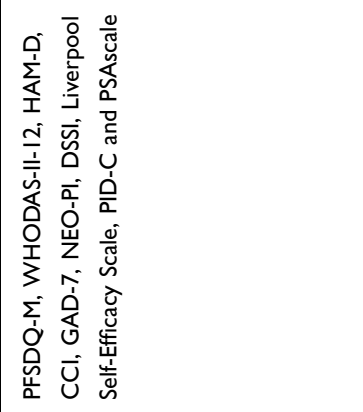 & 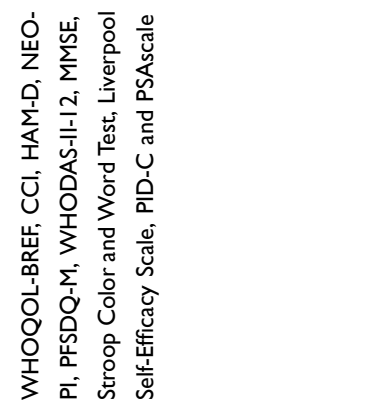 \\
\hline 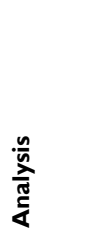 & 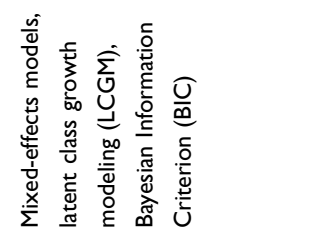 & 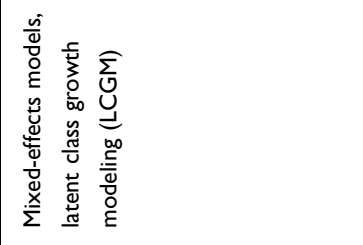 & 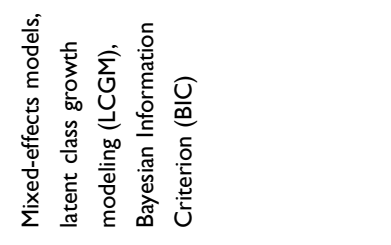 \\
\hline 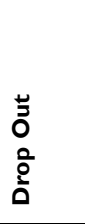 & 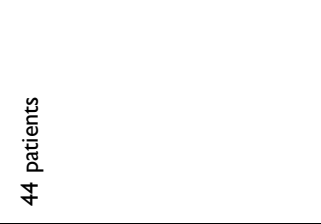 & 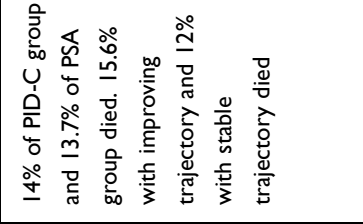 & 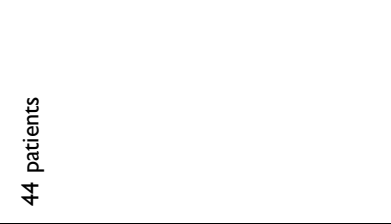 \\
\hline $\begin{array}{l}0 \\
3 \\
3 \\
\overline{0} \\
\dot{0}\end{array}$ & 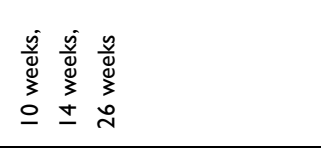 & 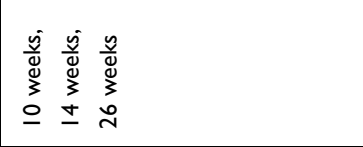 & 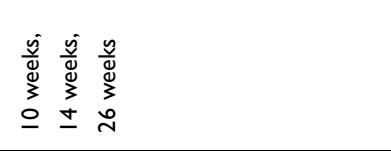 \\
\hline 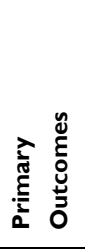 & 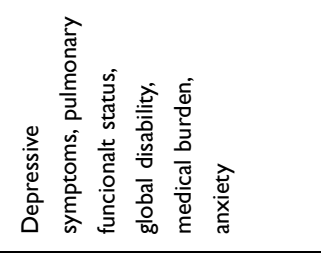 & 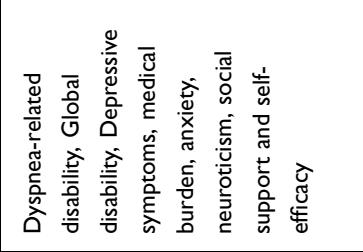 & 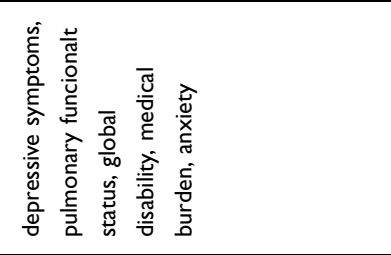 \\
\hline 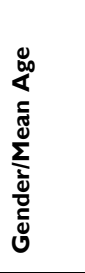 & 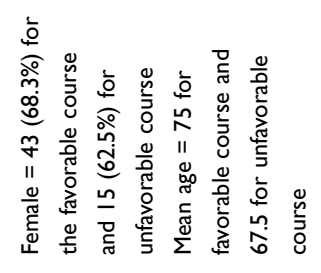 & 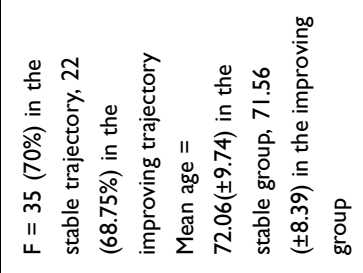 & 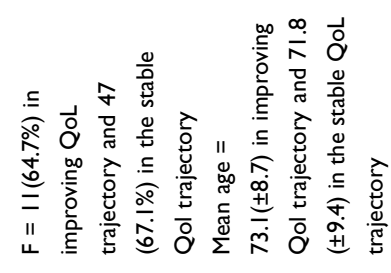 \\
\hline 告 & $\overline{\mathrm{o}}$ & $\overline{\text { ㅇ }}$ & $\overline{\mathrm{o}}$ \\
\hline 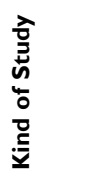 & $\stackrel{t}{\longleftarrow}$ & $\underset{\propto}{\mathfrak{u}}$ & $\underset{\check{u}}{\mathfrak{u}}$ \\
\hline نे & گૅ & 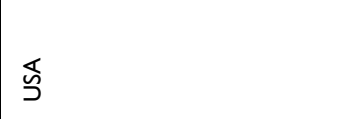 & 芯 \\
\hline 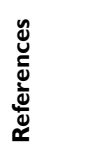 & 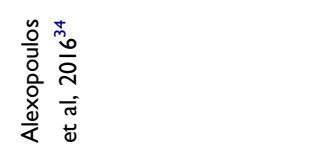 & 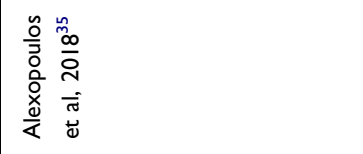 & 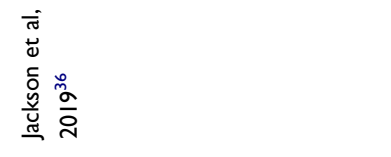 \\
\hline
\end{tabular}




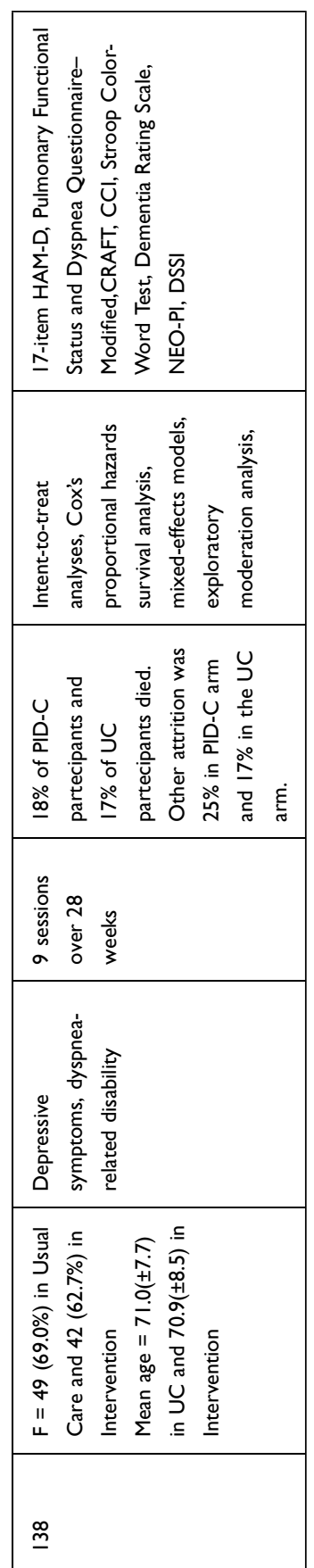

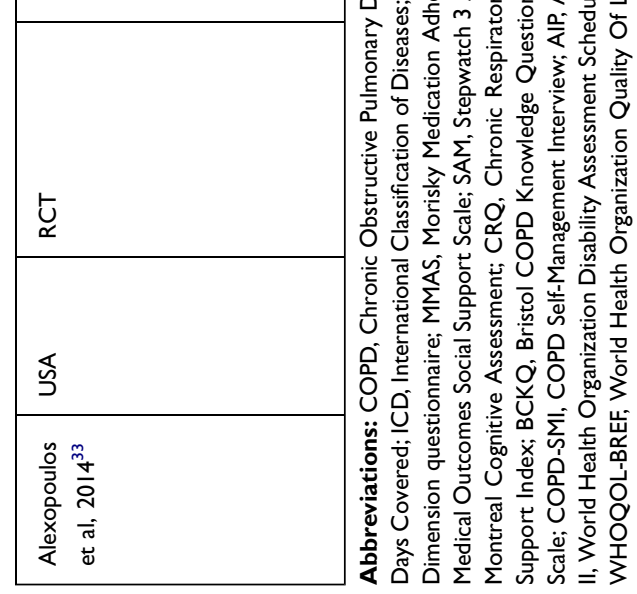

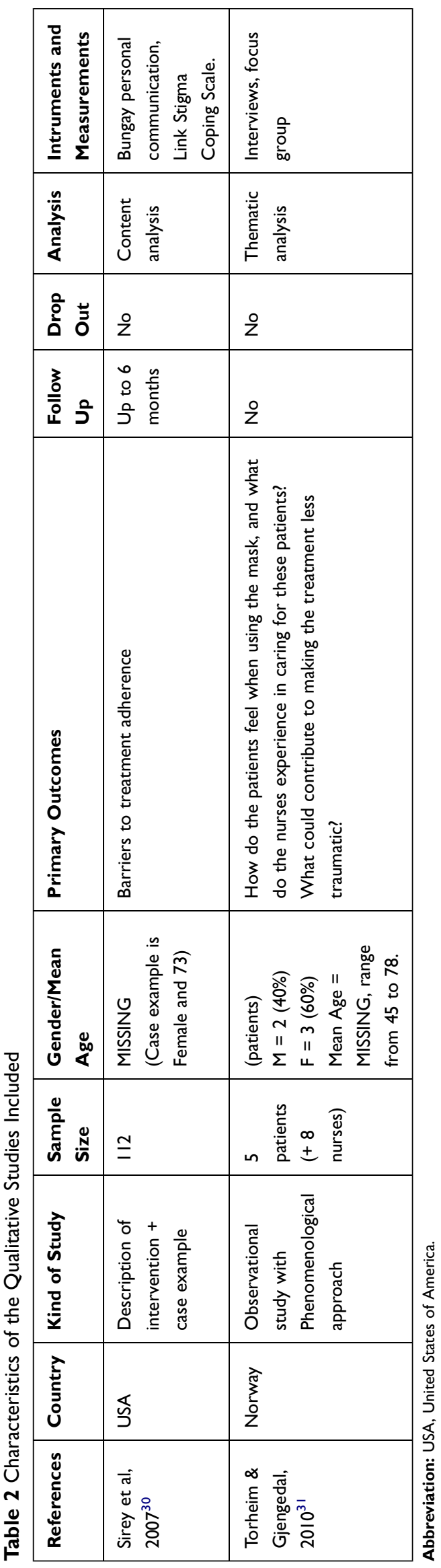




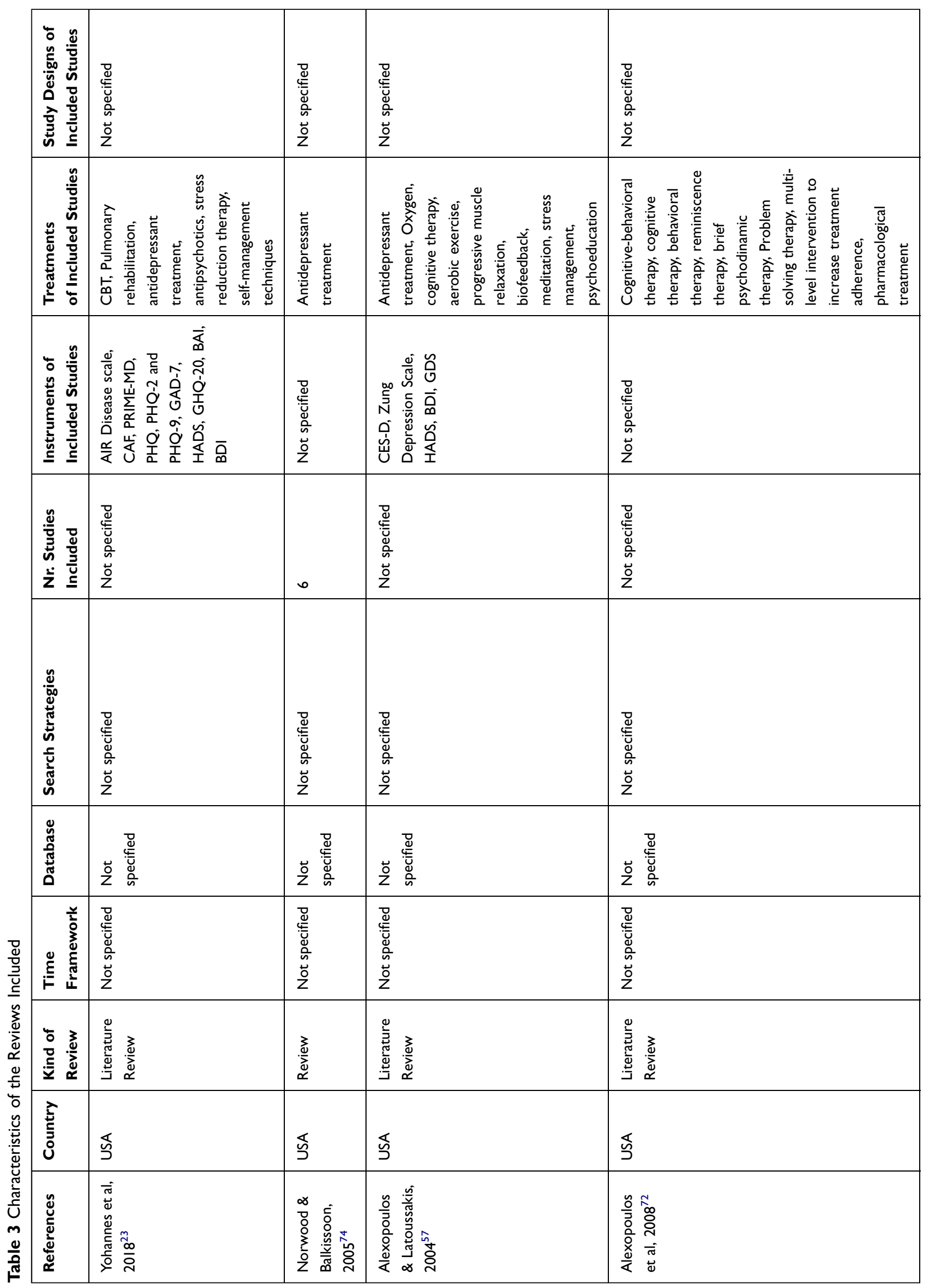




\begin{tabular}{|c|c|c|}
\hline 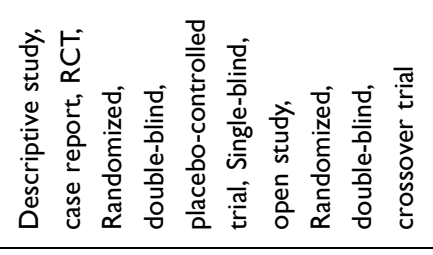 & 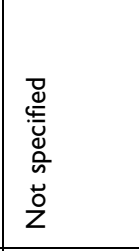 & 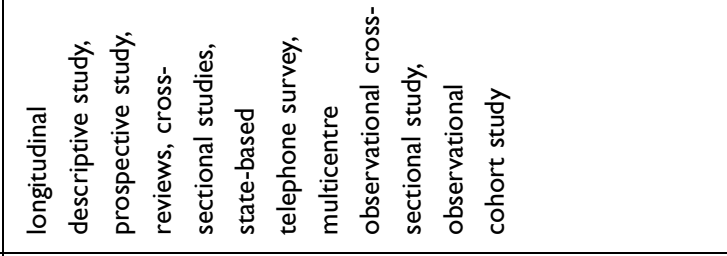 \\
\hline 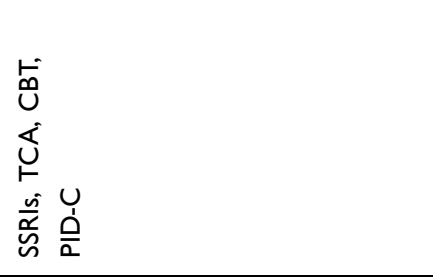 & 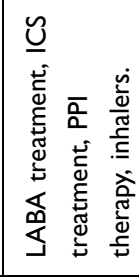 & 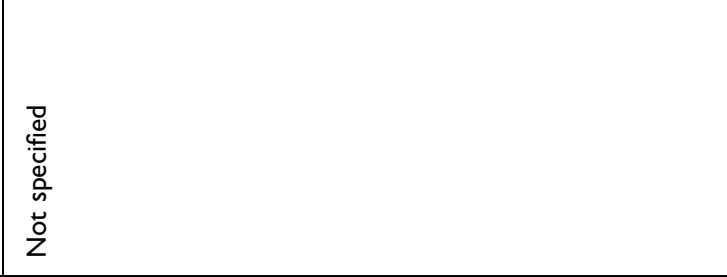 \\
\hline 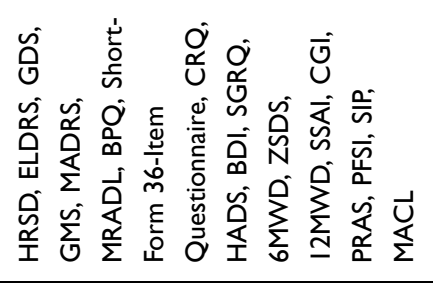 & 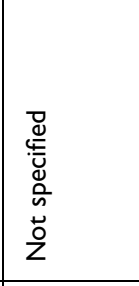 & 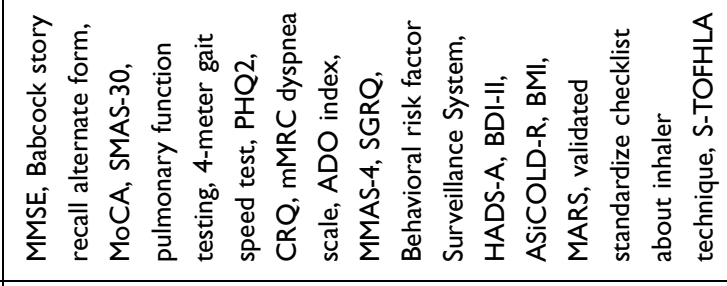 \\
\hline 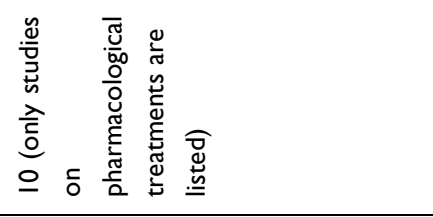 & 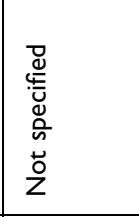 & $\sigma$ \\
\hline 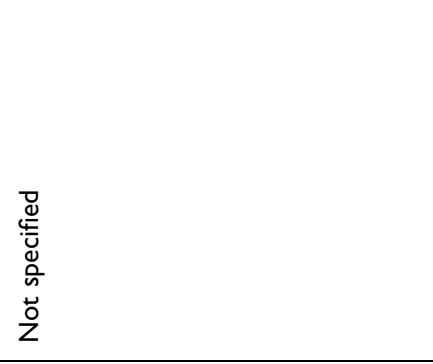 & 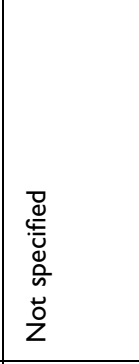 & 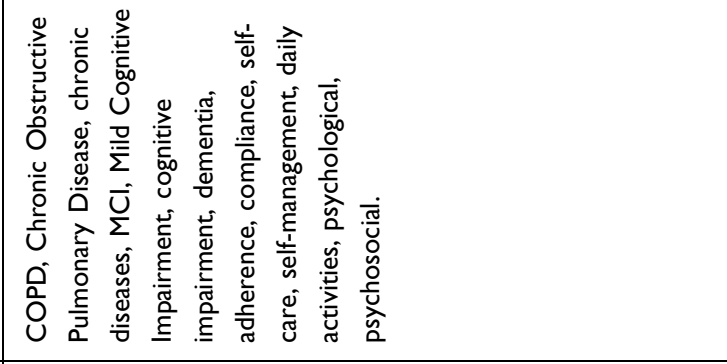 \\
\hline 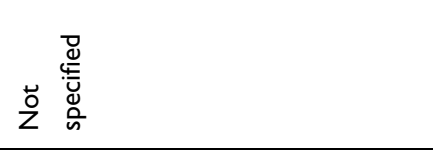 & 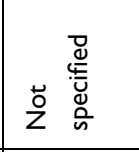 & 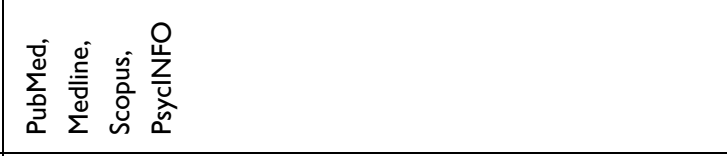 \\
\hline 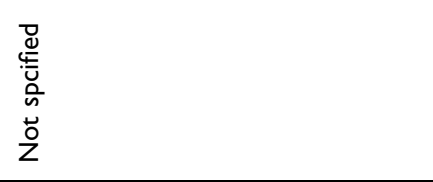 & 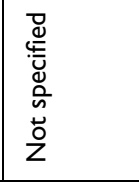 & 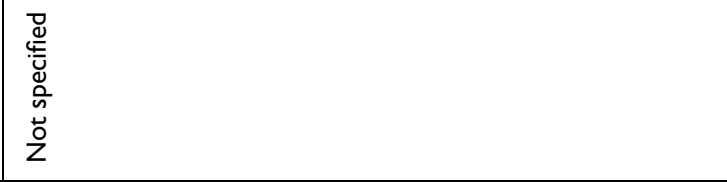 \\
\hline $\begin{array}{l}3 \\
\overline{0} \\
\stackrel{0}{0} \\
\end{array}$ & 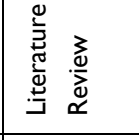 & 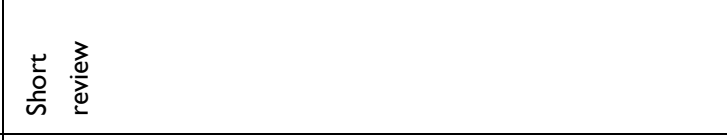 \\
\hline 兰 & 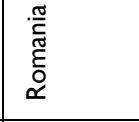 & ब्र \\
\hline 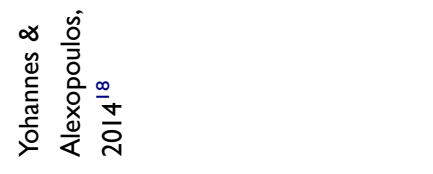 & 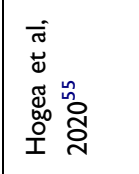 & 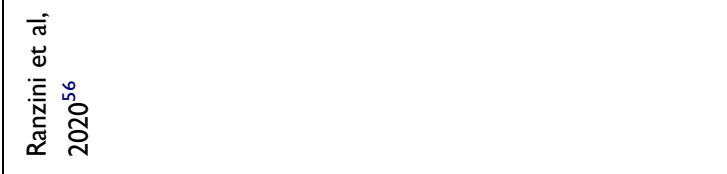 \\
\hline
\end{tabular}


Reviews' sample size (when reported) ranged from 6 studies to 38 studies.

The main characteristics of the included studies are shown in Tables 1-3.

\section{Pathophysiology of Anxiety in COPD and Its Impact on Adherence}

Seven studies reported frequencies of anxiety in their samples. ${ }^{42,46,48-51,53}$ The prevalence varies from a minimum of $23 \%{ }^{51}$ to a maximum of $46 \%{ }^{48}$ Definition of anxiety is not consistent: many studies relied on cut-offs of instruments' scores, though not always specified. For example, a score of 8 or above, ${ }^{25,42,47,48}$ or a score of 11 or above $\mathrm{e}^{45,50}$ in HADS is considered a sign of anxiety. Only a study ${ }^{46}$ discerned between mild $(23.8 \%)$, moderate $(13.1 \%)$, and severe $(3.6 \%)$ anxiety, rather than giving a sum. Other studies reported the mean score of HADS rather than the incidence. Another study $^{49}$ operationalized anxiety in its disorder subtypes according to DSM-IV criteria, with a prevalence of $41 \%$ for panic attacks, $33 \%$ for current general anxiety disorder, and $31 \%$ for anxiety history.

The effect of anxiety on adherence is not clear. The impacts on adherence (or its absence) are rarely considered: for example, Kokturk et $\mathrm{al}^{45}$ measured both anxiety and depression with HADS but examined only the latter. Two studies showed inconsistent results: one ${ }^{48}$ found that adherence was negatively associated with anxiety, while the other ${ }^{46}$ found out that anxiety was associated with an increased adherence. Six studies found no significant association between anxiety and treatment adherence..$^{25,39,42,50,51,53}$ In another study, when considering a scenario of severe COPD exacerbation, patients shown lower action scores (compliance) if they suffered from panic attacks. $^{49}$

As to qualitative studies, Torheim and Gjengedal ${ }^{31}$ identified anxiety as potential comorbidity but did not report the incidence. Anxiety was not measured but identified through thematic analysis in patients' interviews. The focus of this study is not proper adherence but what makes the bi-level positive airway pressure treatment less traumatic (and easier to comply with). Apparently, not knowing how to manage the treatment increased anxiety while regaining control and willpower reduced anxiety.

Only 2 reviews gave specific definition and manifestation examples of anxiety. ${ }^{23,54}$ Yohannes et $\mathrm{al}^{23}$ considered Generalized Anxiety Disorder (GAD), phobias, and panic attacks as "anxiety". Zareifopoulos et $\mathrm{al}^{54}$ only intended
GAD as "anxiety". Prevalence ranges are different: approximately from $10 \%$ to $40 \%,{ }^{23,54}$ from $10 \%$ to $15.8 \%,{ }^{55}$ up to a maximum of $86 \% .{ }^{23}$ Anxiety is often considered comorbidity or a complication of depression. ${ }^{54}$ Indeed, they appeared together in $15.6 \%{ }^{55}$ or $26-43 \%{ }^{23}$ of COPD patients. In the review of Yohannes et al, ${ }^{23}$ anxiety is considered a predictor of poor adherence. In other studies, it is been said that this effect can be due to anxiety lowering self-confidence and selfefficacy $^{55}$ or incrementing cognitive impairment in older patients. $^{56}$ This may increase the risk of COPD exacerbation. ${ }^{54}$ These effects, however, were always considered in combination with depression, as anxiety rarely occurs alone. $^{57}$

\section{Adherence and Anxiety: Measures and Tools}

Many different tools have been used to measure and assess anxiety symptoms related to adherence in COPD, not all of which are validated for use in patients with the condition, or specific or focused enough to truly elicit the required information. The Hospital Anxiety and Depression Scale (HADS), ${ }^{58}$ for instance, is one of the most used to detect both anxiety and depression in the identified clinical studies. Nine studies used the HADS to detect Anxiety. ${ }^{25,39,41,45-48,50,53}$ Turan et al ${ }^{50}$ also used the Anxiety Sensitivity Index (ASI). ${ }^{59}$ Two studies ${ }^{34,35}$ used the Generalized Anxiety Disorder 7-item Scale (GAD7). ${ }^{60}$ Another study ${ }^{33}$ rated anxiety with the sum of psychic and somatic anxiety scores of HAM-D ${ }^{61}$ Some reviews reported other tools. Yohannes et $\mathrm{al}^{23}$ added the Beck Anxiety Inventory (BAI) ${ }^{62}$ the Primary Care Evaluation of Mental Disorders and Patient Health Questionnaire (PRIME-MD; PHQ), ${ }^{63}$ the General Health Questionnaire-version 20 (GHQ-20), ${ }^{64}$ the Anxiety Inventory for Respiratory (AIR) Disease scale ${ }^{65}$ and the COPD Anxiety Questionnaire (CAF) ${ }^{66}$ Only the last two have been specifically developed for respiratory disease. Another review ${ }^{67}$ added the Mood Adjective Checklist (MACL). ${ }^{68}$ Zareifopoulos et $\mathrm{al}^{54}$ added the Perceived Stress Scale (PSS-14), ${ }^{69}$ the EuroQol five-dimension three-level questionnaire (EQ-5D-3L), ${ }^{70}$ and the State-Trait Anxiety Index (STAI). ${ }^{71}$

\section{The Relationship Between Anxiety and Treatment Adherence in COPD: Impact of Interventions}

No interventions aimed to impact anxiety and adherence in COPD patients were found. The relationship between 
interventions, reduction of anxiety, and impact on treatment adherence was not discussed.

\section{Pathophysiology of Depression in COPD and Its Impact on Adherence}

Twelve studies reported frequencies of depression in their samples. $^{30,40,42,43,45,46,48-53}$ The prevalence varies from a minimum of $11 \%^{51}$ to a maximum of $54 \%{ }^{45}$ Depression is often identified through cut-offs of validated scales as HADS or HAM-D. Pierobon et $\mathrm{al}^{46}$ discerned between mild (16.9\%), moderate (15.5\%) and severe (14.3\%) depression according to BDI-II/GDS cut-offs. In other studies, authors used ICD-9-CM codes for depression (excluding bipolar disorder, schizoaffective disorder, and dysthymic disorder) or algorithms based on said codes. $^{37,40,43,44,52}$ Dowson et $\mathrm{al}^{49}$ used DSM-IV criteria. Gauthier et $\mathrm{al}^{51}$ simply noted the previous diagnosis. Other studies reported a mean score of HADS, CED-D, or HAM-D rather than the percentage of depressed patients.

Depression is associated with poorer compliance in most studies, only 3 found no relationship. ${ }^{42,51,53}$ When found, the association between depression and adherence is always negative. In 11 studies ${ }^{25,38-40,43,45-48,50,52}$ higher depression scores or having a diagnosis of depression led to lower adherence. In another study, ${ }^{49}$ depressed patients described themselves as less adherent in severe COPD exacerbation scenarios. According to Jarab and Mukattash, ${ }^{47}$ COPD patients were 4 times more likely to be nonadherent to medications if they reported having depression, in another study, ${ }^{25}$ depressed patients were even 8.9 times more likely to be classified as "nonadherent". Busch et $\mathrm{al}^{38}$ also found out that gender could be a mediator: lower depression predicted higher completion rate or Pulmonary Rehabilitation only for women. Social support seemed to improve compliance in samples with a low HADS score, ${ }^{41}$ but it was not clear if a higher HADS score could decrease this positive effect. Adherence both to COPD and antidepressant pharmacological treatments in depressed patients seemed linked to a decreased risk of hospitalization. ${ }^{37}$ Moreover, depressed patients compliant with an antidepressant were more likely to adherent to COPD maintenance inhalers. $^{44}$

As to qualitative studies, Sirey et $\mathrm{al}^{30}$ declared that the rate of major depression in their sample of depressed COPD patients is $27 \%$.
Only 2 reviews gave specific definition and manifestation examples of depression. ${ }^{23,54}$ Yohannes et $\mathrm{al}^{23}$ considered a major depressive disorder, dysthymia and minor depression as "depression", Zareifopoulos et $\mathrm{al}^{54}$ only intended major depressive disorder as "depression". Four studies provided an incidence of $20 \%$ or more for major depressive disorder in COPD patients. ${ }^{54,57,67,72}$ Prevalence can rise to $40 \%$ for generic depressive symptoms. ${ }^{67}$ Other studies provided ranges from $6 \%$ to $42 \%$ or $50 \%{ }^{55,73,74}$ All reviews identified depression as a barrier to pharmacological and non-pharmacological adherence. Depression can decrease motivation and increase hopelessness, or lower self-esteem, reducing commitment to oxygen masks due to its impact on their appearance. ${ }^{74}$ Depression can lower selfconfidence and self-efficacy ${ }^{55}$ and contribute to the negative effect of cognitive impairment on compliance. ${ }^{56,73}$ The association seemed stronger if the patients have a history of depression and not a recent onset after COPD diagnosis. ${ }^{54}$ On the contrary, improving adherence may reduce depression decreasing dyspnoea-related problems. ${ }^{23}$

\section{Adherence and Depression: Measures and Tools}

Many different tools have been used to assess depression symptoms related to adherence in COPD as well. The $\mathrm{HADS}^{58}$ is used in 8 studies to detect depression. $^{25,39,41,45,47,48,50,53}$ Pierobon et al, ${ }^{46}$ which used the HADS to assess anxiety, assessed depression with the Beck Depression Inventory - second edition (BDI-II) ${ }^{62}$ and the Geriatric Depression Scale (GDS). ${ }^{75}$ Study $^{38}$ employed the Center for Epidemiologic Studies Depression Scale (CES-D). ${ }^{76}$ Another tool is the Hamilton Rating Scale for Depression (HRSD or HAM-D), ${ }^{61}$ in different versions. ${ }^{32-36}$ One of these studies ${ }^{34}$ also used the SCID-R Interview. ${ }^{77}$

Reviews reported other tools in addition. Yohannes et $\mathrm{al}^{23}$ appointed PRIME-MD and PHQ, ${ }^{63}$ along with HADS and BDI. Alexopoulos and Latoussakis ${ }^{57}$ added the Zung Self-Rating Depression Scale (ZSDS) ${ }^{78}$ and specified that the HADS, though the most used scale, may tend to overdiagnose depression disorders in COPD patients. Another review ${ }^{67}$ added the Evans Liverpool Depression Rating Scale (ELDRS), ${ }^{79}$ the Geriatric Mental State (GMS) Examination, ${ }^{80}$ the Montgomery-Asberg Depression Rating Scale (MADRS), ${ }^{81}$ and the MACL. ${ }^{68}$ Zareifopoulos et $\mathrm{al}^{54}$ added the EQ-5D-3L. ${ }^{70}$ 


\section{The Relationship Between Depression and Treatment Adherence in COPD: Impact of Interventions}

Sirey et $\mathrm{al}^{30}$ illustrated a multi-level intervention aimed to improve treatment adherence in COPD patients. The intervention, led by a care manager, included an interview before discharge in which barriers to treatments were identified, and follow-up sessions at home. Negative beliefs about depression and antidepressants and the perceived stigma toward depressed people or COPD patients hurt adherence: assessing these issues to create a personalized treatment led to better clinical outcomes.

This review includes a series of RCTs assessing the efficacy of adherence-based interventions in depressed COPD patients: PID-C (Personalized Intervention for Depression and COPD) and PSA (Problem Solving Adherence). The assumption was that depression, disability, and poor adherence interact in COPD patients; thus, an approach targeting these variables is needed. ${ }^{72}$ PIC-D identifies treatment barriers and helps patients to improve adherence - as the intervention of Sirey et $\mathrm{al}^{30}-$ and, compared to Treatment as Usual (TAU), led to a significative greater reduction of depressive symptoms, and dyspnoea-related disability. ${ }^{32,33}$ The PSA program, including problem-solving skills to improve adherence, was designed to be more efficient than PIC-D. However, the hypothesis was not supported: PSA indeed improved depression, quality of life and dyspnoea-related disability, but not more than PID-C. ${ }^{34-36}$ This said, interventions assessing treatment adherence seemed to be effective in reducing depression and breaking the "vicious cycle".

\section{Measurements and Definition of Adherence}

Adherence was assessed in many ways (Table 4). Five studies calculated compliance with Proportion of Days Covered. ${ }^{37,40,43,44,52}$ Other authors choose medical measurements like the concentrator's counter clock to measure the daily duration of oxygen, ${ }^{51}$ the number of doses taken/the number of doses prescribed X $100^{42}$ or adherence to $>80 \%$ of pharmacological dosage prescribed. ${ }^{33}$ To Busch et al, "adherence" was the completion of at least 20 sessions of pulmonary rehabilitation. Other researchers $^{53}$ asked coordinators to provide information on whether respondents dropped out of rehabilitation or not. The Morisky Medication Adherence Scale (MMAS), ${ }^{82}$ a validated scale for adherence, was employed in four studies ${ }^{25,45-47}$ and cited in two reviews. $^{56,73}$ One of those ${ }^{46}$ also used the Adherence Schedule in Chronic Obstructive Lung Disease Revised (ASiCOLD-R). ${ }^{83}$ Study $^{48}$ employed a short COPD Action Plan developed by the Family Physician Airway Group of Canadian Thoracic Society. ${ }^{84}$ In other cases, self-reported questionnaires or interviews were developed for the intervention. ${ }^{34-36,39,41,49}$ Turan et $\mathrm{al}^{50}$ scored the usage of bronchodilator according to the "National Guide of Turkish Thoracic Society for Asthma". ${ }^{85}$ One study did not specify how adherence was measured. ${ }^{32}$ In qualitative studies, ${ }^{30,31}$ adherence was not objectively measured but assessed during interviews and extrapolated in thematic analysis. One review ${ }^{73}$ was focused on adherence and reported other scales: the Medication Adherence Report Scale (MARS), ${ }^{86}$ the Measure of Treatment Adherence (MTA), ${ }^{87}$ and the Test of Adherence to Inhaler (TAI). ${ }^{88}$ This review is also the only article reporting a definition of adherence, as "the extent to which the person's behavior corresponds with the agreed recommendations from a health care provider". 73

It must be noted that the included studies considered adherence concerning different kinds of treatments. Some studies $^{25,37,40,43-45,47,50,52}$ and a review ${ }^{73}$ considered compliance to pharmacological prescriptions and inhaled therapy such as LABAs (Long-acting $\beta$ adrenoceptor agonists), LAMAs (Long-acting muscarinic antagonist), and ICS (inhaled corticosteroid). Three studies considered Pulmonary Rehabilitation or maintenance exercise programs after PR. ${ }^{38,39,53}$ Other studies considered adherence to LTOT, ${ }^{51}$ BPAP mask treatment, ${ }^{31}$ or domiciliary nebulized therapy. ${ }^{42}$ Two studies considered both pharmacological and nonpharmacological prescriptions, like PR attendance, smoking, exercise, etc. ${ }^{41,46}$ In other research, adherence is operationalized as a generic sum of various medical and rehabilitation recommendations. ${ }^{30,48,49}$ In all the RCTs, the adherence enhanced by the PID-C or the PSA interventions is a generic "adherence" to physician recommendations. ${ }^{32-36}$

\section{Confounding Factors and Methodological Issues in Studies}

A relevant methodological issue is the high risk of bias of some of the included studies, especially in qualitative studies and reviews. Seven studies had a low risk of bias, 18 studies had a medium risk of bias, 9 studies ( 7 
Table 4 List of Included Studies That Discussed Different Kinds of Adherence

\begin{tabular}{|c|c|}
\hline Article & Adherence to \\
\hline Albrecht et al, $2017^{37}$ & $\begin{array}{l}\text { Antidepressants, COPD maintenance medications (corticosteroids, long-acting } \beta \text {-agonists, long-acting } \\
\text { anticholinergics), healthcare utilization }\end{array}$ \\
\hline $\begin{array}{l}\text { Heerema-Poelman et al, } \\
2013^{39}\end{array}$ & Maintenance exercise program after pulmonary rehabilitation \\
\hline Zucchelli et al, $2020^{40}$ & Triple inhaled therapy (with different devices) \\
\hline Kokturk et al, $2018^{45}$ & Oxygen, pharmacological treatment, inhalators \\
\hline Gauthier et al, $2018^{51}$ & LTOT \\
\hline Albrecht et al, $2016^{43}$ & COPD maintenance medication (inhaled corticosteroids, long-acting $\beta$-agonists, long-acting anticholinergics) \\
\hline Sirey et al, $2007^{30}$ & Generic psychiatric, medical and rehabilitation recommendations \\
\hline Qian et al, $2014^{52}$ & $\begin{array}{l}\text { COPD maintenance medication (inhaled corticosteroids, long-acting } \beta \text {-agonists, ICS/LABA combinations, long-acting } \\
\text { anticholinergics, methylxanthines) }\end{array}$ \\
\hline Chen et al, $2017^{41}$ & "self care behaviour" (pulmonary rehabilitation attendance, medication adherence, smoking cessation) \\
\hline Pierobon et al, $2017^{46}$ & Pharmacological adherence, nonpharmacological prescriptions (eating, smoking, exercise, LTOT) \\
\hline Jarab \& Mukattash, $2019^{47}$ & $\begin{array}{l}\text { COPD maintenance medication (short-acting } \beta 2 \text {-agonist, long-acting } \beta \text {-agonists, long-acting anticholinergics, oral } \\
\text { steroids, antibiotics) }\end{array}$ \\
\hline $\begin{array}{l}\text { Torheim \& Gjengedal, } \\
2009^{31}\end{array}$ & BPAP mask treatment \\
\hline Doyle et al, $2013^{53}$ & Pulmonary rehabilitation \\
\hline Choi et al, $2014^{48}$ & Generic COPD Action Plan (including usage of meds or oxygen, avoiding triggers, contacting healthcare providers) \\
\hline Alexopoulos et al, $2013^{32}$ & Generic adherence to COPD meds and recommendations (enhanced by the PID-C intervention) \\
\hline Kdhour et al, $2012^{25}$ & Medications \\
\hline Bosley et al, $1996^{42}$ & Domiciliar nebulized therapy \\
\hline Dowson et al, $2004^{49}$ & Generic actions to self-management and maintenance \\
\hline Wei et al, $2018^{44}$ & Maintenance medications (oxygen, rescue/acute meds), antidepressants \\
\hline Turan et al, $2014^{50}$ & Bronchodilator therapy \\
\hline Alexopoulos et al, $2016^{34}$ & Generic adherence to physicians' recommendations (enhanced by the PID-C and the PSA interventions) \\
\hline Alexopoulos et al, $2018^{35}$ & Generic adherence to physicians' recommendations (enhanced by the PID-C and the PSA interventions) \\
\hline Jackson et al, $2019^{36}$ & Generic adherence to physicians' recommendations (enhanced by the PID-C and the PSA interventions) \\
\hline Alexopoulos et al, $2014^{33}$ & Generic adherence to COPD meds and recommendations (enhanced by the PID-C intervention) \\
\hline Busch et al, $2014^{38}$ & Pulmonary Rehabilitation \\
\hline
\end{tabular}

Abbreviations: COPD, Chronic Obstructive Pulmonary Disease; LTOT, long-Term Oxygen Therapy; ICS, Inhaled corticosteroids; LABA, Long-acting beta-agonists; PID-C, Personalized Intervention for Depression and COPD; PSA, Problem Solving Adherence.

of which are reviews) had a high risk of bias. In a qualitative study, ${ }^{30}$ the methodology and the research design were not clear, as recruitment criteria, and lacked a description of data analysis. Plus, findings were partially stated. Reviews were unfortunately hardly valuable too, being mostly narrative and literature reviews and not systematic. The reviews with the lowest risk of bias met $6 / 8^{73}$ and $5 / 8^{54}$ criteria of the quality assessment tool, the other 7 reviews had a high risk of bias, one of them met no criteria. $^{23}$ Often, the focus question or aim was 
formulated, but eligibility criteria, search strategy, time framework, and databases were missing. Only 3 reviews listed the articles included. ${ }^{67,73,74}$ Moreover, reviews were included if they had at least a paragraph focused on the link between depression/anxiety and compliance, but none of these studies were directly aimed to study this relationship. In observational and cohort studies, the risk of bias was mostly medium. Studies with the lowest risk of bias met $10 / 14$ criteria of the NIH quality assessment tool. ${ }^{37,41,44}$ The study with the highest risk of bias met $4 /$ 14 criteria: $:^{53}$ the sample was not well described, the loss to follow-up was $>20 \%$ and much information was missing, such as $\mathrm{r}$ - or p-value in statistical analysis. Other studies were overall well described, but with small samples and no sample size justification, or high attrition. In other cases, exposures were assessed just once, and some data were missing. The inconsistency between studies in depression and anxiety measurements and adherence definition and the variety of different treatments and interventions included could be a confounding factor for generalizability.

Among the five RCTs included in this review, three had a low risk of bias, and two showed some concerns. One was just a brief report of the experiment, and few details were available. ${ }^{32}$ Another had a small sample, with a huge drop-out before randomization, and the same therapist administrated both experimental, and control interventions. ${ }^{36}$ In the remaining studies, the limitations like high attrition were discussed by researchers and possible solutions were provided (Appendix C).

\section{Discussion}

This review evaluates the state of the literature on the relationship between anxiety, depression, and compliance in COPD patients. To our knowledge, the existing literature lacks systematic reviews specifically focused on this topic. Mood disorders can lead to a vicious cycle, reducing selfconfidence, increasing isolation and aggravating dyspnoearelated disability, and reducing adherence to pharmacological and non-pharmacological treatments. ${ }^{54}$ The potential impact of psychological issues on adherence in COPD treatment (despite the discussed limitations) should be taken into consideration by physicians and properly evaluated when COPD is diagnosed, not only for their impact on adherence but also on mortality, disability, and quality of life. Findings regarding depression were clearer and more consistent, leading to the conclusion that this mood disorder (especially when severe) could probably have a negative influence on treatment adherence, direct ${ }^{23,57,73}$ or mediated. ${ }^{55,56,73,74}$ However, different measurements and definitions of depression and operationalization of adherence could have been confounding factors. Findings concerning anxiety, on the other hand, were less coherent. The literature lacks studies about the impact of anxiety on adherence and their relationships. This fact elicits a doubt on the methodological value of research instruments and designs. The number of studies with a high or medium risk of bias was surely a limitation, especially among reviews. These kinds of publications are useful to frame and understand a phenomenon, but they are not sufficient to systematically observe the state of literature about a topic. Moreover, included reviews had at least a paragraph focused on the link between depression/anxiety and compliance, but none of these studies were directly aimed to study this relationship. Another limitation was the discrepancy between the focus on anxiety and depression: many studies assessed the impact of depressive symptoms, but the anxiety was not highlighted in the same way. In many reviews and quantitative studies, anxiety was only mentioned or measured but not included in statistical models. No research question was aimed to evaluate the impact of anxiety alone, its effect was rather considered in combination with depression, and the lack of studies focused on this variable proves the need for further evaluations. "Adherence" was operationalized in different ways across studies, through validated scales, interviews with physicians or arbitrary cut-offs depending on treatments. This issue was addressed in one of the included reviews: the authors underlined the discrepancies in the definitions and measurement criteria of adherence, possibly leading to variances in nonadherence rates. ${ }^{73}$ Moreover, the treatment of reference could be pharmacological or non-pharmacological, LTOT, pulmonary rehabilitation, exercise programs, and so on. Future research should investigate if the inconsistency could be due to a different kinds of "adherence" or evaluate if the different ways to measure depression and anxiety have an impact on results. Moreover, the type of connection between these variables should be clearer: in some cases, researchers investigate a correlation, in other cases a regression model. Different research designs have different implications when evaluating the results. As to Randomized Controlled Trials, not every RCT assessed the direct impact of adherence improvement on depression, but they all pertained to the same set of experiments, carried out by the same research group, showing that adherence-based interventions helped COPD depressed patients in different ways, directly and indirectly. However, some studies are the same experiment, 
thus the hypothesis should be investigated by further research, evaluate the specific efficacy of an adherencebased treatment on depression, and maybe on anxiety as well. At the review-level, we cannot ignore the possible influence of publication bias and selective reporting bias: undesirable, incoherent, or not significative results may be underrepresented in literature due to the selection carried out by researchers and journals. Eligible studies could be also partially retrieved, due to other uncontrollable biases. The involvement of three researchers in study evaluation and data extraction should have reduced the bias during the selection phase, however, the risk is not eliminated.

\section{Conclusions}

This systematic review suggests that the relationship between depression and adherence should be considered relevant during the implementation of a personalized treatment plan. On the other hand, the relationship between anxiety and adherence in COPD is still controversial. Moreover, there is a need for further exploration of this phenomenon with a comprehensive, systematic approach, considering if there is a difference between different instruments to measure anxiety and depression and different ways to operationalize adherence. The development of models comprehensive of both anxiety and depression concerning adherence with clinically useful levels of accuracy, sensitivity, and specificity has not yet been achieved.

Future research should evaluate the impact of depression and anxiety, taken both alone and together, finding the common features between these variables and how these features influence compliance, distinguishing between different types of treatment adherence.

\section{Data Sharing Statement}

All relevant data are within the paper and its Supporting Information files.

\section{Author Contributions}

EV and FP conceived of the present idea. EV, ST and FP performed investigation, data curation, formal analysis and methodology. PB and FP supervised the review development. ST and EV wrote the first draft and then, reviewed and edited it according to the considerations of PB and FP. All authors made substantial contributions to conception and design, acquisition of data, or analysis and interpretation of data; took part in drafting the article or revising it critically for important intellectual content; agreed to submit to the current journal; gave final approval of the version to be published; and agree to be accountable for all aspects of the work. Francesco Pagnini is a co-senior author.

\section{Funding}

This work was not supported by any funder.

\section{Disclosure}

The authors have declared no conflicts of interest for this work.

\section{References}

1. Vogelmeier CF, Criner GJ, Martinez FJ, et al. Global strategy for the diagnosis, management, and prevention of chronic obstructive lung disease 2017 report. Am J Respir Crit Care Med. 2017;195 (5):557-582. doi:10.1164/rccm.201701-0218PP

2. Panagioti M, Scott C, Blakemore A, Coventry PA. Overview of the prevalence, impact, and management of depression and anxiety in chronic obstructive pulmonary disease. Int $J$ COPD. 2014;9:1289-1306. doi:10.2147/COPD.S72073

3. Berry CE, Wise RA. Mortality in COPD: causes, risk factors, and prevention. COPD J Chronic Obstr Pulm Dis. 2010;7(5):375-382. doi: $10.3109 / 15412555.2010 .510160$

4. Yohannes AM, Baldwin RC, Connolly MJ. Depression and anxiety in elderly outpatients with chronic obstructive pulmonary disease: prevalence, and validation of the BASDEC screening questionnaire. Int J Geriatr Psychiatry. 2000;15(12):1090-1096. doi:10.1002/ 1099-1166(200012)15:12<090::aid-gps249>3.0.CO;2-L

5. Kunik ME, Roundy K, Veazey C, et al. Surprisingly high prevalence of anxiety and depression in chronic breathing disorders. Chest. 2005;127(4):1205-1211. doi:10.1378/chest.127.4.1205

6. Phan T, Carter O, Waterer G, et al. Determinants for concomitant anxiety and depression in people living with chronic obstructive pulmonary disease. J Psychosom Res. 2019;120:60-65. doi:10.1016/ j.jpsychores.2019.03.004

7. Biswas D, Mukherjee S, Chakroborty R, et al. Occurrence of anxiety and depression among stable COPD patients and its impact on functional capability. J Clin Diagnostic Res. 2017;11(2):OC24-OC27. doi:10.7860/JCDR/2017/24203.9393

8. Tetikkurt C, Ozdemir I, Tetikkurt S, Yilmaz N, Ertan T, Bayar N. Anxiety and depression in COPD patients and correlation with sputum and BAL cytology. Multidiscip Respir Med. 2011;6(4):226-231. doi:10.1186/2049-6958-6-4-226

9. Willgoss TG, Yohannes AM. Anxiety disorders in patients with COPD: a systematic review. Respir Care. 2013;58(5):858-866. doi: $10.4187 /$ respcare. 01862

10. Maurer J, Rebbapragada V, Borson S, et al. Anxiety and depression in COPD: current understanding, unanswered questions, and research needs. Chest. 2008;134(4 suppl):43S-56S. doi:10.1378/chest.08-0342

11. Dowson C, Laing R, Barraclough R, et al. The use of the Hospital Anxiety and Depression Scale (HADS) in patients with chronic obstructive pulmonary disease: a pilot study. $N Z$ Med $J$. 2001;114 (1141):447-449.

12. Yohannes AM, Willgoss TG, Baldwin RC, Connolly MJ. Depression and anxiety in chronic heart failure and chronic obstructive pulmonary disease: prevalence, relevance, clinical implications and management principles. Int J Geriatr Psychiatry. 2010;25(12):1209-1221. doi: $10.1002 /$ gps. 2463

13. Laurin C, Moullec G, Bacon SL, Lavoie KL. Impact of anxiety and depression on chronic obstructive pulmonary disease exacerbation risk. Am J Respir Crit Care Med. 2012;185(9):918-923. doi:10.1164/rccm.201105-0939PP 
14. Ng TP, Niti M, Tan WC, Cao Z, Ong KC, Eng P. Depressive symptoms and chronic obstructive pulmonary disease: effect on mortality, hospital readmission, symptom burden, functional status, and quality of life. Arch Intern Med. 2007;167(1):60-67. doi:10.1001/ archinte.167.1.60

15. Atlantis E, Fahey P, Cochrane B, Smith S. Bidirectional associations between clinically relevant depression or anxiety and COPD: a systematic review and meta-analysis. Chest. 2013;144 (3):766-777. doi:10.1378/chest.12-1911

16. Giardino ND, Curtis JL, Andrei AC, et al. Anxiety is associated with diminished exercise performance and quality of life in severe emphysema: a cross-sectional study. Respir Res. 2010;11(1):29. doi:10.1186/1465-9921-11-29

17. Blakemore A, Dickens C, Guthrie E, et al. Depression and anxiety predict health-related quality of life in chronic obstructive pulmonary disease: systematic review and meta-analysis. Int $J$ COPD. 2014;9:501-512. doi:10.2147/COPD.S58136

18. Yohannes AM, Alexopoulos GS. Depression and anxiety in patients with COPD. Eur Respir Rev. 2014;23(133):345-349. doi:10.1183/ 09059180.00007813

19. Sundh J, Ekström M. Persistent disabling breathlessness in chronic obstructive pulmonary disease. Int J COPD. 2016;11(1):2805-2812. doi:10.2147/COPD.S119992

20. Yohannes AM, Müllerová H, Hanania NA, et al. Long-term course of depression trajectories in patients with COPD: a 3-year follow-up analysis of the evaluation of COPD longitudinally to identify predictive surrogate endpoints cohort. Chest. 2016;149(4):916-926. doi:10.1016/j.chest.2015.10.081

21. Burgel. PR, Escamilla R, Perez T, et al. Impact of comorbidities on COPD-specific health-related quality of life. Respir Med. 2013;107 (2):233-241.

22. Von Leupoldt A, Kenn K. The psychology of chronic obstructive pulmonary disease. Curr Opin Psychiatry. 2013;26(5):458-463. doi:10.1097/YCO.0b013e328363c1fc

23. Yohannes AM, Kaplan A, Hanania NA. Anxiety and depression in chronic obstructive pulmonary disease: recognition and management. Cleveland Clin J Med. 2018;85(2 Suppl 1):S11-S18.

24. Fan VS, Giardino ND, Blough DK, et al. Costs of pulmonary rehabilitation and predictors of adherence in the National Emphysema Treatment Trial. COPD J Chronic Obstr Pulm Dis. 2008;5 (2):105-116. doi:10.1080/15412550801941190

25. Khdour MR, Hawwa AF, Kidney JC, Smyth BM, McElnay JC. Potential risk factors for medication non-adherence in patients with chronic obstructive pulmonary disease (COPD). Eur $J$ Clin Pharmacol. 2012;68(10):1365-1373. doi:10.1007/s00228-012-1279-5

26. Wang KY, Sung PY, Yang ST, Chiang CH, Perng WC. Influence of family caregiver caring behavior on COPD Patients' self-care behavior in Taiwan. Respir Care. 2012;57(2):263-272. doi:10.4187/respcare.00986

27. DiMatteo MR, Lepper HS, Croghan TW. Depression is a risk factor for noncompliance with medical treatment meta-analysis of the effects of anxiety and depression on patient adherence. Arch Intern Med. 2000;160(2101):2107. doi:10.1001/archinte.160.14.2101

28. Moher D, Liberati A, Tetzlaff J, Altman DG, PRISMA group. Preferred reporting items for systematic reviews and meta-analyses: the PRISMA statement. PLoS Med. 2009;6(7):e1000097. doi:10.1371/journal.pmed.1000097

29. Sterne JAC, Savović J, Page MJ, et al. RoB 2: a revised tool for assessing risk of bias in randomised trials. BMJ. 2019;366. doi:10.1136/bmj.14898

30. Sirey JA, Raue PJ, Alexopoulos GS. An intervention to improve depression care in older adults with COPD. Int $J$ Geriatr Psychiatry. 2007;22(2):154-159. doi:10.1002/gps.1705

31. Torheim H, Gjengedal E. How to cope with the mask? Experiences of mask treatment in patients with acute chronic obstructive pulmonary disease-exacerbations. Scand J Caring Sci. 2010;24(3):499-506. doi:10.1111/j.1471-6712.2009.00740.x
32. Alexopoulos GS, Kiosses DN, Sirey JA, et al. Personalised intervention for people with depression and severe COPD. Br J Psychiatry. 2013;202(3):235-236. doi:10.1192/bjp.bp.112.120139

33. Alexopoulos GS, Kiosses DN, Sirey JA, et al. Untangling therapeutic ingredients of a personalized intervention for patients with depression and severe COPD. Am J Geriatr Psychiatry. 2014;22(11):1316-1324. doi:10.1016/j.jagp.2013.05.006

34. Alexopoulos GS, Sirey JA, Banerjee S, et al. Two behavioral interventions for patients with major depression and severe COPD. Am $J \quad$ Geriatr Psychiatry. 2016;24(11):964-974. doi:10.1016/j. jagp.2016.07.014

35. Alexopoulos GS, Sirey JA, Banerjee S, et al. Two interventions for patients with major depression and severe chronic obstructive pulmonary disease: impact on dyspnea-related disability. Am J Geriatr Psychiatry. 2018;26(2):162-171. doi:10.1016/j.jagp.2017.10.002

36. Jackson DS, Banerjee S, Sirey JA, et al. Two interventions for patients with major depression and severe chronic obstructive pulmonary disease: impact on quality of life. Am J Geriatr Psychiatry. 2019;27(5):502-511. doi:10.1016/j.jagp.2018.12.004

37. Albrecht JS, Khokhar B, Huang TY, et al. Adherence and healthcare utilization among older adults with COPD and depression. Respir Med. 2017;129:53-58. doi:10.1016/j.rmed.2017.06.002

38. Busch AM, Scott-Sheldon LAJ, Pierce J, et al. Depressed mood predicts pulmonary rehabilitation completion among women, but not men. Respir Med. 2014;108(7):1007-1013. doi:10.1016/j. rmed.2014.04.010

39. Heerema-Poelman A, Stuive I, Wempe JB. Adherence to a maintenance exercise program 1 year after pulmonary rehabilitation: what are the predictors of dropout? J Cardiopulm Rehabil Prev. 2013;33(6):419-426. doi:10.1097/HCR.0b013e3182a5274a

40. Zucchelli A, Vetrano DL, Bianchini E, et al. Adherence to COPD free triple inhaled therapy in the real world: a primary care based study. Clin Respir J. 2020;14(8):732-739. doi:10.1111/crj.13190

41. Chen Z, Fan VS, Belza B, Pike K, Nguyen HQ. Association between social support and self-care behaviors in adults with chronic obstructive pulmonary disease. Ann Am Thorac Soc. 2017;14(9):1419-1427. doi:10.1513/AnnalsATS.201701-026OC

42. Bosley CM, Corden ZM, Rees PJ, Cochrane GM. Psychological factors associated with use of home nebulized therapy for COPD. Eur Respir J. 1996;9(11):2346-2350. doi:10.1183/09031936.9 6.09112346

43. Albrecht JS, Park Y, Hur P, et al. Adherence to maintenance medications among older adults with chronic obstructive pulmonary disease the role of depression. Ann Am Thorac Soc. 2016;13(9):1497-1504. doi:10.1513/AnnalsATS.201602-136OC

44. Wei YJ, Simoni-Wastila L, Albrecht JS, et al. The association of antidepressant treatment with COPD maintenance medication use and adherence in a comorbid Medicare population: a longitudinal cohort study. Int J Geriatr Psychiatry. 2018;33(2):e212-e220. doi:10.1002/ gps.4772

45. Kokturk N, Polatli M, Oguzulgen IK, et al. Adherence to COPD treatment in turkey and Saudi Arabia: results of the ADCARE study. Int J COPD. 2018;13:1377-1388. doi:10.2147/COPD.S150411

46. Pierobon A, Bottelli ES, Ranzini L, et al. COPD patients' selfreported adherence, psychosocial factors and mild cognitive impairment in pulmonary rehabilitation. Int J COPD. 2017;12:2059-2067. doi:10.2147/COPD.S133586

47. Jarab AS, Mukattash TL. Exploring variables associated with medication non-adherence in patients with COPD. Int J Clin Pharm. 2019;41(5):1202-1209. doi:10.1007/s11096-019-00895-3

48. Choi JY, Chung HIC, Han G. Patient outcomes according to COPD action plan adherence. J Clin Nurs. 2014;23(5-6):883-891. doi:10.1111/jocn.12293

49. Dowson CA, Town GI, Frampton C, Mulder RT. Psychopathology and illness beliefs influence COPD self-management. J Psychosom Res. 2004;56(3):333-340. doi:10.1016/S0022-3999(03)00040-0 
50. Turan O, Yemez B, Itil O. The effects of anxiety and depression symptoms on treatment adherence in COPD patients. Prim Health Care Res Dev. 2014;15(3):244-251. doi:10.1017/ S1463423613000169

51. Gauthier A, Bernard S, Bernard E, Simard S, Maltais F, Lacasse Y. Adherence to long-term oxygen therapy in patients with chronic obstructive pulmonary disease. Chron Respir Dis. 2018;16:1479972318767724. doi:10.1177/1479972318767724

52. Qian J, Simoni-Wastila L, Rattinger GB, et al. Association between depression and maintenance medication adherence among Medicare beneficiaries with chronic obstructive pulmonary disease. Int J Geriatr Psychiatry. 2014;29(1):49-57. doi:10.1002/gps.3968

53. Doyle C, Dunt D, Ames D, Selvarajah S. Managing mood disorders in patients attending pulmonary rehabilitation clinics. Int $J$ COPD. 2013;8:15-20. doi:10.2147/COPD.S36378

54. Zareifopoulos N, Bellou A, Spiropoulou A, Spiropoulos K. Prevalence, contribution to disease burden and management of comorbid depression and anxiety in chronic obstructive pulmonary disease: a narrative review. COPD J Chronic Obstr Pulm Dis. 2019;16(5-6):406-417. doi:10.1080/15412555.2019.1679102

55. Hogea SP, Tudorache E, Fildan AP, Fira-Mladinescu O, Marc M, Oancea C. Risk factors of chronic obstructive pulmonary disease exacerbations. Clin Respir J. 2020;14(3):183-197. doi:10.1111/ crj. 13129

56. Ranzini L, Schiavi M, Pierobon A, Granata N, Giardini A. From Mild Cognitive Impairment (MCI) to Dementia in Chronic Obstructive Pulmonary Disease. Implications for clinical practice and disease management: a mini-review. Front Psychol. 2020;11(February):1-6. doi:10.3389/fpsyg.2020.00337

57. Alexopoulos GS, Latoussakis V. Depression comorbidity with COPD. Psychiatr Ann. 2004;34(4):289-296. doi:10.3928/00485713-20040401-09

58. Zigmond AS, Snaith RP. The hospital anxiety and depression scale. Acta Psychiatr Scand. 1983;67(6):361-370. doi:10.1111/j.16000447.1983.tb09716.x

59. Reiss S, Peterson RA, Gursky DM, McNally RJ. Anxiety sensitivity, anxiety frequency and the prediction of fearfulness. Behav Res Ther. 1986;24:1-8. doi:10.1016/0005-7967(86)90143-9

60. Spitzer RL, Kroenke K, Williams JBW, Löwe B. A brief measure for assessing generalized anxiety disorder: the GAD-7. Arch Intern Med. 2006;166(10):1092-1097. doi:10.1001/archinte.166.10.1092

61. Hamilton M. A rating scale for depression. J Neurol Neurosurg Psychiatry. 1960;23(1):56-62. doi:10.1136/jnnp.23.1.56

62. Beck AT, Steer RA, Brown GK. Manual for the Beck Depression Inventory-II. Vols. 1-82. San Antonio, TX: Psychol Corp; 1996.

63. Spitzer RL, Kroenke K, Williams JB. Validation and utility of a self-report version of PRIME-MD: the PHQ primary care study. Primary Care Evaluation of Mental Disorders. Patient Health Questionnaire. JAMA. 1999;282(18):1737-1744.

64. Goldberg DP. The detection of psychiatric illness by questionnaire; a technique for the identi-fication and assessment of non-psychotic illness. Can Psychiatr Assoc J. 1972;18(4):348-349.

65. Willgoss TG, Goldbart J, Fatoye F, Yohannes AM. The development and validation of the anxiety inventory for respiratory disease. Chest. 2013;144(5):1587-1596. doi:10.1378/chest.13-0168

66. Kühl K, Kuhn C, Kenn K, Rief W. The COPD-anxiety-questionnaire (CAF): a new instrument to assess illness specific anxiety in COPD patients. PPmP - Psychother - Psychosom - Medizinische Psychol. 2011;61(1):e1-9. doi:10.1055/s-0030-1248281

67. Yohannes AM, Alexopoulos GS. Pharmacological treatment of depression in older patients with chronic obstructive pulmonary disease: impact on the course of the disease and health outcomes. Drugs Aging. 2014;31(7):483-492. doi:10.1007/s40266-014-0186-0

68. Matthews G, Jones DM, Chamberlain AG. Refining the measurement of mood: the UWIST Mood Adjective Checklist. Br J Psychol. 1990;81(1):17-42. doi:10.1111/j.2044-8295.1990.tb02343.x
69. Cohen S, Kamarck T, Mermelstein R. A global measure of perceived stress. J Health Soc Behav. 1983;24:385-396. doi:10.2307/2136404

70. Rabin R, De Charro F. EQ-5D: a measure of health status from the EuroQol Group. Ann Med. 2001;33:337-343. doi:10.3109/ 07853890109002087

71. Spielberger CD, Gorsuch RL, Lushene RE. STAI Manual for the State-Trait Anxiety Inventory. Self-Evaluation Questionnaire. Palo Alto, CA: Consulting Psychologists Press; 1970.

72. Alexopoulos GS, Raue PJ, Sirey JA, Arean PA. Developing an intervention for depressed, chronically medically ill elders: a model from COPD. Int $J$ Geriatr Psychiatry. 2008;23(5):447-453. doi: $10.1002 /$ gps. 1925

73. Bhattarai B, Walpola R, Mey A, Anoopkumar-Dukie S, Khan S. Barriers and strategies for improving medication adherence among people living with COPD: a systematic review. Respir Care. 2020;65 (11):1738-1750. doi:10.4187/respcare. 07355

74. Norwood R, Balkissoon R. Current perspectives on management of co-morbid depression in COPD. COPD J Chronic Obstr Pulm Dis. 2005;2(1):185-193. doi:10.1081/COPD-200050740

75. Yesavage JA, Brink TL, Rose TL, et al. Development and validation of a geriatric depression screening scale: a preliminary report. $J$ Psychiatr Res. 1982;17(1):37-49. doi:10.1016/0022-3956(82) 90033-4

76. Radloff LS. The CES-D scale: a self-report depression scale for research in the general population. Appl Psychol Meas. 1977;1:385-401. doi:10.1177/014662167700100306

77. Spitzer RL, Williams J, Gibbon W, First MB. User's guide for the Structured Clinical Interview for DSM-IIIR (SCID). Am Psychiatr. 1990;272:1749-1756.

78. Zung WWK. A self-rating depression scale. Arch Gen Psychiatry. 1965;12(1):63-70. doi:10.1001/archpsyc.1965.01720310065008

79. Evans ME. Development and validation of a brief screening scale for depression in the elderly physically ill. Int Clin Psychopharmacol. 1993;8(4):329-331. doi:10.1097/00004850-199300840-00022

80. Copeland JR, Dewey ME, Griffiths-Jones HM. A computerized psychiatric diagnostic system and case nomenclature for elderly subjects: GMS and AGECAT. Psychol Med. 1986;16(1):89-99. doi:10.1017/ S0033291700057779

81. Montgomery SA, Asberg M. A new depression scale designed to be sensitive to change. Br J Psychiatry. 1979;134:382-389. doi:10.1192/ bjp.134.4.382

82. Morisky DE, Green LW, Levine DM. Concurrent and predictive validity of a self-reported measure of medication adherence. Med Care. 1986;24:67-74. doi:10.1097/00005650-198601000-00007

83. Majani G, Pierobon A, Giardini A, Callegari S. Valutare e Favorire L'aderenza Alle Prescrizioni in Riabilitazione Cardiologica e Pneumologica. (Maugeri IM Della F, Ed.). Pime; 2007.

84. Kaplan A. The COPD action plan. Can Fam Physician. 2009;55:58-59.

85. Van Der Palen J, Klein JJ, Van Herwaarden CLA, Zielhuis GA, Seydel ER. Multiple inhalers confuse asthma patients. Eur Respir J. 1999;14(5):1034-1037. doi:10.1183/09031936.99.14510349

86. Horne R, Weinman J. Self-regulation and self-management in asthma: exploring the role of illness perceptions and treatment beliefs in explaining non-adherence to preventer medication. Psychol Health. 2002;17(1):17-32.

87. Delgado A, Lima M. Contributo para a validação concorrente de uma medidade de adesão aos tratamentos. Psicol Saúde Doenças. 2001;2:81-100.

88. Plaza V, Fernández-Rodríguez $\mathrm{C}$, Melero $\mathrm{C}$, et al. Validation of the "Test of the Adherence to Inhalers" (TAI) for Asthma and COPD Patients. J Aerosol Med Pulm Drug Deliv. 2016;29(2):142-152. doi:10.1089/jamp.2015.1212 


\section{Publish your work in this journal}

The International Journal of COPD is an international, peer-reviewed journal of therapeutics and pharmacology focusing on concise rapid reporting of clinical studies and reviews in COPD. Special focus is given to the pathophysiological processes underlying the disease, intervention programs, patient focused education, and self management

protocols. This journal is indexed on PubMed Central, MedLine and CAS. The manuscript management system is completely online and includes a very quick and fair peer-review system, which is all easy to use. Visit http://www.dovepress.com/testimonials.php to read real quotes from published authors.

Submit your manuscript here: https://www.dovepress.com/international-journal-of-chronic-obstructive-pulmonary-disease-journal 